Delft University of Technology

\title{
MSE-impact of PPP-RTK ZTD estimation strategies
}

Wang, K.; Khodabandeh, A.; Teunissen, P. J.G.

DOI

10.1016/j.asr.2018.04.012

Publication date

2018

Document Version

Final published version

Published in

Advances in Space Research

\section{Citation (APA)}

Wang, K., Khodabandeh, A., \& Teunissen, P. J. G. (2018). MSE-impact of PPP-RTK ZTD estimation strategies. Advances in Space Research, 61(12), 2955-2971. https://doi.org/10.1016/j.asr.2018.04.012

\section{Important note}

To cite this publication, please use the final published version (if applicable).

Please check the document version above.

\section{Copyright}

Other than for strictly personal use, it is not permitted to download, forward or distribute the text or part of it, without the consent of the author(s) and/or copyright holder(s), unless the work is under an open content license such as Creative Commons.

\section{Takedown policy}

Please contact us and provide details if you believe this document breaches copyrights.

We will remove access to the work immediately and investigate your claim. 
Green Open Access added to TU Delft Institutional Repository 'You share, we take care!' - Taverne project

\section{https://www.openaccess.nl/en/you-share-we-take-care}

Otherwise as indicated in the copyright section: the publisher is the copyright holder of this work and the author uses the Dutch legislation to make this work public. 


\title{
MSE-impact of PPP-RTK ZTD estimation strategies
}

\author{
K. Wang ${ }^{\text {a }}$, A. Khodabandeh ${ }^{\mathrm{a}, *}$, P.J.G. Teunissen ${ }^{\mathrm{a}, \mathrm{b}}$ \\ ${ }^{a}$ GNSS Research Centre, Curtin University of Technology, GPO Box U1987, Perth, WA 6845, Australia \\ ${ }^{\mathrm{b}}$ Department of Geoscience and Remote Sensing, Delft University of Technology, 2628 CN Delft, The Netherlands
}

Received 21 December 2017; received in revised form 14 March 2018; accepted 12 April 2018

Available online 22 April 2018

\begin{abstract}
In PPP-RTK network processing, the wet component of the zenith tropospheric delay (ZTD) cannot be precisely modelled and thus remains unknown in the observation equations. For small networks, the tropospheric mapping functions of different stations to a given satellite are almost equal to each other, thereby causing a near rank-deficiency between the ZTDs and satellite clocks. The stated near rank-deficiency can be solved by estimating the wet ZTD components relatively to that of the reference receiver, while the wet ZTD component of the reference receiver is constrained to zero. However, by increasing network scale and humidity around the reference receiver, enlarged mismodelled effects could bias the network and the user solutions. To consider both the influences of the noise and the biases, the mean-squared errors (MSEs) of different network and user parameters are studied analytically employing both the ZTD estimation strategies. We conclude that for a certain set of parameters, the difference in their MSE structures using both strategies is only driven by the square of the reference wet ZTD component and the formal variance of its solution. Depending on the network scale and the humidity condition around the reference receiver, the ZTD estimation strategy that delivers more accurate solutions might be different. Simulations are performed to illustrate the conclusions made by analytical studies. We find that estimating the ZTDs relatively in large networks and humid regions (for the reference receiver) could significantly degrade the network ambiguity success rates. Using ambiguity-fixed network-derived PPP-RTK corrections, for networks with an inter-station distance within $100 \mathrm{~km}$, the choices of the ZTD estimation strategy is not crucial for single-epoch ambiguity-fixed user positioning. Using ambiguity-float network corrections, for networks with inter-station distances of 100, 300 and $500 \mathrm{~km}$ in humid regions (for the reference receiver), the root-mean-squared errors (RMSEs) of the estimated user coordinates using relative ZTD estimation could be higher than those under the absolute case with differences up to millimetres, centimetres and decimetres, respectively.
\end{abstract}

(C) 2018 COSPAR. Published by Elsevier Ltd. All rights reserved.

Keywords: Zenith Tropospheric Delay (ZTD); PPP-RTK; Mean-Squared Error (MSE); Near rank-deficiency; Mismodelled effects

\section{Introduction}

The zenith tropospheric delay (ZTD), which is multiplied by an elevation-dependent mapping function, is one of the typical unknowns in GNSS observation equations (Hofmann-Wellenhof et al., 2008; Teunissen and Montenbruck, 2017). The hydrostatic component of the

\footnotetext{
* Corresponding author.

E-mail addresses: kan.wang@curtin.edu.au (K. Wang), amir khodabandeh@curtin.edu.au (A. Khodabandeh), p.teunissen@curtin. edu.au (P.J.G. Teunissen).
}

tropospheric delay, which reaches around $2.3 \mathrm{~m}$ in the zenith direction and is mainly related to the temperature and air pressure, varies smoothly and slowly in time and can be precisely modelled with mm-accuracy or even better based on surface meteorological data (Bevis et al., 1992; Wang and $\mathrm{Li}, 2016)$. In the zenith direction, the wet component of the tropospheric delay varies from centimetres (or less) in the arid regions to as large as $35 \mathrm{~cm}$ in the humid regions (Bevis et al., 1992; Younes, 2016). It is mainly related to the water vapour and is difficult to be modelled with high accuracy, since water vapour is not a 
well-mixed constituent of the atmosphere (Resch, 1984). As a result, the estimation of the wet component of the ZTDs is important in high-precision GNSS applications (Mousa et al., 2016; Rothacher and Beutler, 1998).

The PPP-RTK technique, introduced by Wübbena et al. (2005), is also known as integer ambiguity resolution enabled Precise Point Positioning (PPP). In the last ten years, diverse studies were performed in this area (Collins, 2008; Ge et al., 2008; Geng and Shi, 2017; Laurichesse and Mercier, 2007; Loyer et al., 2012; Mervart et al., 2008; Teunissen et al., 2010) with a review given in Teunissen and Khodabandeh (2015). In PPPRTK processing, in order to avoid singularities in the design matrix, estimable parameters are formed based on the $S$-system theory (Baarda, 1981; Teunissen, 1985a). For small networks, the tropospheric mapping functions of different receivers to a given satellite are almost equal to each other due to their almost identical elevation angles to this satellite (Odijk et al., 2014b; Khodabandeh and Teunissen, 2015). As a result, an additional near rankdeficiency exists in the design matrix between the columns for the estimable ZTDs and satellite clocks (Odijk et al., 2012). To solve this problem, instead of estimating the wet ZTD components of each receiver, referred to as "absolute" ZTD estimation in this contribution, the wet ZTD component of the reference receiver can be constrained. The estimable ZTD parameters would then take between-receiver forms, referred to as "relative" ZTDs in this contribution (Odijk et al., 2011, 2012, 2014b; Teunissen and Montenbruck, 2017).

With the increasing scale of the network, however, the assumption that the tropospheric mapping functions of different stations to the same satellite are almost equal does not hold anymore. The unignorable difference in the tropospheric mapping functions leads to mismodelled effect in case of relative ZTD estimation, which is related to both the wet ZTD component of the reference receiver and the between-receiver difference of the tropospheric mapping functions. Depending on the network scale and the humidity condition around the reference receiver, the mismodelled effects could bias the network and the user solutions in different manners. Since the mean-squared error (MSE) describes both the influences of the noise and the mismodelled effect on the estimated parameters, it is used in this contribution to evaluate the accuracies of the network and user solutions. With the ZTDs estimated absolutely and relatively, the MSEs of different sets of network and user parameters could response differently to the network scale and the wet ZTD component of the reference receiver. In this contribution, we first analytically compute and compare the MSEs of different network and user parameters employing both ZTD estimation strategies. The conclusions are then illustrated with numerical results based on simulation studies using networks with different scales and under different humidity conditions around the reference receiver. In this contribution, for a certain set of PPP-RTK network and user parameters, we aim to show that the difference in their MSE structures using both ZTD estimation strategies is only driven by two components, i.e., the square of the reference wet ZTD component and the formal variance of its solution. Depending on the scale of the network, the humidity condition around the reference receiver as well as the processing time, comparison of the square roots of these two components directly gives us the ZTD estimation strategy that delivers smaller root-mean-squared errors (RMSEs) of these parameters.

In Section 2, we firstly explain our processing procedure in terms of linear algebra and apply it to the network and the user part of the PPP-RTK processing. The strategies of absolute and relative ZTD estimation are explained in detail, and the MSEs of different sets of network and user parameters are derived using both ZTD estimation strategies. With the settings of the network and the user processing introduced, numerical results based on simulation studies are discussed in Section 3. Simulated networks in Australia consisting of 3 stations with an inter-station distance varying from 1 to $500 \mathrm{~km}$ are used for the computation, and a wet ZTD component of the reference receiver varying from 0 to $3.5 \mathrm{dm}$ is pre-defined to simulate different humidity conditions around the reference receiver. Using both ZTD estimation strategies, the RMSEs are evaluated and compared in Section 3.1 for networks with different scales and under different humidity conditions around the reference receiver. The comparison is also performed for the ambiguity success rates (ASRs) based on simulated float ambiguities considering also the mismodelled effects for relative ZTD estimation. In Section 3.2, the RMSEs of the estimated user coordinates are computed and compared using network corrections under both ZTD estimation strategies. Two cases are discussed with respect to the RMSE comparison, i.e., using the ambiguity-fixed and -float network corrections. For each of these two cases, the choices of the ZTD estimation strategy that supplies more accurate user coordinate estimates are discussed for networks with different scales and under different humidity conditions around the reference receiver. The conclusions are given in Section 4.

We use the following notation throughout this contribution. $\mathrm{E}($.$) and \mathrm{D}($.$) represent the expectation and dispersion$ operators, respectively. The operator $\operatorname{tr}($.$) denotes the trace$ of a matrix. An estimator of parameter $x$ is indicated by the

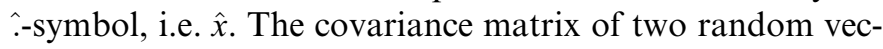
tors $\hat{x}$ and $\hat{y}$ is symbolized by $Q_{\hat{x} \hat{y}}$. Thus $\mathrm{D}(\hat{x})=Q_{\hat{x} \hat{x}}$. The MSE and RMSE of the random vector $\hat{x}$ are denoted by $\operatorname{MSE}(\hat{x})$ and $\operatorname{RMSE}(\hat{x})$, respectively.

\section{Near rank-deficiency of GNSS observation equations}

\subsection{Near-singular linear models}

In the following we discuss the three-component structure of PPP-RTK (Mervart et al., 2008; Teunissen et al., 
2010; Wübbena et al., 2005) in the context of linear algebra. The stated structure is composed of (1) networkcomponent, (2) correction component and (3) usercomponent.

\subsubsection{Network-component}

As our point of departure we commence with the network observation equations expressed by the following linear model

$\mathrm{E}(y)=A x$

and its known dispersion

$\mathrm{D}(y)=Q_{y y}$

with $y$ and $x$ being the observation and parameter vectors, respectively. The variance matrix $Q_{y y}$ is positive definite and the known design matrix $A$ is of full-column rank. Thus the parameter vector $x$ (and any linear function thereof) are assumed to be estimable under model (1). Let us now further assume that there exists a nonzero vector, say $v$, for which

$\epsilon=A v$

represents a vector of small values, i.e. $\epsilon \approx 0$. This implies that columns of $A$ are almost linearly dependent, thereby leaving functions of $x$ poorly estimable. To characterize such functions, consider an arbitrary full-column rank matrix $S$ whose columns, together with $v$, form a square and invertible transformation matrix $[v, S]$. The parameter vector $x$ can then be expressed in terms of its transformed versions $\alpha$ and $\beta$ through the one-toone transformation

$x=v \beta+S \alpha \quad \Longleftrightarrow \quad[v, S]^{-1} x=\left[\beta^{T}, \alpha^{T}\right]^{T}$

Substitution into the network model (1), together with (3), gives

$\mathrm{E}(y)=\epsilon \beta+A S \alpha$

The parameter $\beta$ is thus weakly linked to the observation vector $y$, as the corresponding column vector $\epsilon$ is small. As a consequence, the variance of its Best Linear Unbiased Estimator (BLUE) $\hat{\beta}$ is large and can be shown to be bounded from below as follows (Teunissen, 1985b)

$\sigma_{\hat{\beta}}^{2}=\frac{1}{\bar{\epsilon}^{T} Q_{y y}^{-1} \bar{\epsilon}} \geqslant \frac{1}{\epsilon^{T} Q_{y y}^{-1} \boldsymbol{\epsilon}}$

where

$\overline{\boldsymbol{\epsilon}}=\boldsymbol{\epsilon}-A S\left(S^{T} N S\right)^{-1} S^{T} N v, \quad$ with $\quad N=A^{T} Q_{y y}^{-1} A$

According to (6), the smaller the squared-norm $\|\epsilon\|_{Q_{v y}}^{2}=\epsilon^{T} Q_{y y}^{-1} \epsilon$, the larger the lower bound of the variance $\sigma_{\hat{\beta}}^{2}$ becomes. In the extreme case when $\epsilon \rightarrow 0$, we have $\sigma_{\hat{\beta}}^{2} \rightarrow \infty$, i.e. $\beta$ becomes completely inestimable. Therefore, any linear function of $\alpha$ combined with $\beta$ is also poorly estimable under the assumption $\epsilon \approx 0$.
We now turn our attention to functions of $x$ which do not depend on $\beta$. Such functions, say $z$, can be formed by eliminating the column vector $v$ in (4). Thus with $L$ being a basis matrix of the null space of $v^{T}$, i.e. $v^{T} L=0$, the stated functions can be characterized as follows (cf. (4))

$z=L^{T} x=L^{T} S \alpha$

Since $z$ does not depend on the poorly estimable parameter $\beta$, its BLUE $\hat{z}$ is expected to have finite variances. We are therefore interested to study the 'accuracy' of $\hat{z}$ under the following two scenarios:

- Scenario A: The unknown parameter $\beta$, in (5), is kept as unknown and is to be estimated together with the $\alpha$ parameters. The corresponding solution of $z$ is denoted by $\hat{z}_{A}$.

- Scenario B: The unknown parameter $\beta$, in (5), is constrained to be zero, i.e. $\beta=0$. The corresponding solution of $z$ is denoted by $\hat{z}_{B}$.

On the one hand, Scenario A delivers the network solution $\hat{z}_{A}$ that is unbiased, i.e. $\mathrm{E}\left(\hat{z}_{A}\right)=z$, but it may represent low precision due to the inclusion of the additional unknown $\beta$. One the other hand, Scenario B delivers the network solution $\hat{z}_{B}$ that has a better precision-level, but it becomes biased when $\beta \neq 0$, i.e., $\mathrm{E}\left(\hat{z}_{B}\right) \neq z$. One may then use the MSE criterion to measure the solutions' accuracy, thereby considering both the 'precision' and 'bias' of $\hat{z}_{A}$ and $\hat{z}_{B}$. The following lemma shows how the MSEs of the stated solutions are related.

Lemma 1 (MSEs of $\hat{z}_{A}$ and $\hat{z}_{B}$ ). Consider the network observation Eq. (5) and the unknown parameters $z$ given in (8). Let $\hat{z}_{A}$ and $\hat{z}_{B}$ be the BLUEs of $z$ under Scenarios $A$ and $B$, respectively. They follow as weighted least-squares solutions of $z$ where the inverse variance matrix $Q_{y y}^{-1}$ is taken as weight matrix. The MSEs of $\hat{z}_{A}$ and $\hat{z}_{B}$ can then be given as

Scenario A : $\quad \operatorname{MSE}\left(\hat{z}_{A}\right):=\mathrm{E}\left\|\hat{z}_{A}-z\right\|^{2}=\operatorname{tr}\left(Q_{\hat{z}_{B} \hat{z}_{B}}\right)+\sigma_{\hat{\beta}}^{2} h^{T} h$

Scenario B : $\quad \operatorname{MSE}\left(\hat{z}_{B}\right):=\mathrm{E}\left\|\hat{z}_{B}-z\right\|^{2}=\operatorname{tr}\left(Q_{\hat{z}_{B} \hat{z}_{B}}\right)+\beta^{2} h^{T} h$

with $h=Q_{\hat{z}_{B} \hat{y}_{B}} Q_{y y}^{-1} \epsilon$, where $Q_{{\hat{\hat{B}_{B}}}_{B}}$ and $Q_{\hat{z}_{B} \hat{y}_{B}}$ are the variance matrix of $\hat{z}_{B}$ and the covariance matrix between $\hat{z}_{B}$ and the adjusted observation $\hat{y}_{B}$ under Scenario B, respectively.

\section{Proof. see Appendix A.}

Note the similarity between the MSEs of $\hat{z}_{A}$ and $\hat{z}_{B}$ in (9). Their difference in structure is only driven by the difference between the two scalars: the variance $\sigma_{\hat{\beta}}^{2}$ and the squared bias $\beta^{2}$. When $\beta=0$, the solution $\hat{z}_{B}$ outperforms its counterpart $\hat{z}_{A}$ in the MSE sense, i.e. the MSE of $\hat{z}_{B}$ becomes smaller than that of $\hat{z}_{A}$. This is, however, not the case when $\beta^{2}>\sigma_{\hat{\beta}}^{2}$. In that case, Scenario A delivers better solutions in the MSE sense. Note also, in contrast to the variance $\sigma_{\hat{\beta}}^{2}$ 
that becomes very large when $\epsilon$ tends to zero, that the MSE of $\hat{z}_{A}$ remains finite. This is due to the presence of the term $h^{T} h$ serving as the multiplier of $\sigma_{\hat{\beta}}^{2}$. When $\epsilon$ tends to zero, the term $h^{T} h$ becomes very small so that the product $\sigma_{\hat{\beta}}^{2} h^{T} h$ remains finite. In Section 2.2 we will show Scenarios A and B at work, where a GNSS network model serves as an example of (5).

\subsubsection{Correction-component}

Not all the network parameters $z$ and $\beta$ involved in (5) are of interest to PPP-RTK users. Apart from orbital corrections, the user only needs to be provided with satellitespecific corrections (i.e. clock and biases) and (sometimes) atmospheric corrections. Let such corrections, denoted by $c$, be given as

$c=F^{T} z+b \beta$

Thus the known coefficient matrix $F$ and vector $b$ form the corrections $c$ as functions of the network parameters $z$ and $\beta$. The BLUEs of the corrections (10), under Scenarios $\mathrm{A}$ and $\mathrm{B}$, read

Scenario A : $\quad \hat{c}_{A}=F^{T} \hat{z}_{A}+b \hat{\beta}$

Scenario B : $\quad \hat{c}_{B}=F^{T} \hat{z}_{B}, \quad(\hat{\beta}=0)$

Note that the solution $\hat{c}_{A}$ is unbiased, i.e. $\mathrm{E}\left(\hat{c}_{A}\right)=c$, since $\mathrm{E}\left(\hat{z}_{A}\right)=z$ and $\mathrm{E}(\hat{\beta})=\beta$. The solution $\hat{c}_{B}$ is, however, biased when $\beta \neq 0$, i.e. $\mathrm{E}\left(\hat{c}_{B}\right) \neq c$. The stated bias is given by

$$
\begin{aligned}
\mathrm{E}\left(\hat{c}_{B}\right)-c & =\mathrm{E}\left(\hat{c}_{B}-\hat{c}_{A}\right) \\
& =F^{T} \mathrm{E}\left(\hat{z}_{B}-\hat{z}_{A}\right)-b \mathrm{E}(\hat{\beta}) \\
& =\left(F^{T} h-b\right) \beta
\end{aligned}
$$

where the third equality follows from $\mathrm{E}\left(\hat{z}_{B}\right)=\mathrm{E}\left(\hat{z}_{A}\right)+h \beta$ (cf. Appendix A). Now the question is how the unaccounted bias $\left(F^{T} h-b\right) \beta$ affects the MSE performance of PPP-RTK user solutions. This will be addressed in the following.

\subsubsection{User-component}

Let $y_{u}$ be the user observation vector, having the positive definite variance matrix $Q_{y_{u} y_{u}}$. The user aims to determine the unknown parameter vector $x_{u}$ that is linked to the observations $y_{u}$ through the known full-column rank design matrix $A_{u}$. The user observation equations, however, contains extra unknown parameters which make the user model 'rank-deficient' (i.e. not all the unknowns can be determined by the user observations). Such rank-deficient user observation equations read

$\mathrm{E}\left(y_{u}\right)=A_{u} x_{u}-\underbrace{\left(F^{T} z+b \beta\right)}_{c}$

with $\mathrm{D}\left(y_{u}\right)=Q_{y_{u} y_{u}}$. Thus the extra parameters are nothing else but the network corrections $c$ that have to be a priori provided to the user. Adding the corrections (11) to $y_{u}$ gives the corrected user observation equations as follows

Scenario A : $\quad \mathrm{E}\left(y_{u}+\hat{c}_{A}\right)=A_{u} x_{u}$

Scenario B : $\quad \mathrm{E}\left(y_{u}+\hat{c}_{B}\right)=A_{u} x_{u}+\left[\left(F^{T} h-b\right) \beta\right]$

As shown, the bias of the corrections $\hat{c}_{B}$ (cf. (12)) shows up as a 'mismodelled' effect in the corrected user observation equations under Scenario B. It is a 'mismodelled' effect as the user does not consider them to be present in the model, thereby remaining unaccounted for.

In practice, the user is not often provided with the variance matrix of the corrections $\hat{c}_{A}$ and $\hat{c}_{B}$. The stated corrections are then treated as if they are 'non-random'. As a consequence, the user takes the inverse variance matrix $Q_{y_{u} y_{u}}^{-1}$ as weight matrix to compute weighted least-squares solutions of $x_{u}$. Such solutions, say $\hat{x}_{u_{A}}$ and $\hat{x}_{u_{B}}$, do therefore not represent the BLUEs of $x_{u}$, but just its weighted leastsquares solutions. Under Scenario A, the least-squares solution $\hat{x}_{u_{A}}$ is unbiased, i.e. $\mathrm{E}\left(\hat{x}_{u_{A}}\right)=x_{u}$. The precision of $\hat{x}_{u_{A}}$ might, however, be adversely affected by the presence of the poorly precise solution $\hat{\beta}$. Under Scenario B, the least-squares solution $\hat{x}_{u_{B}}$ is not affected by the variance $\sigma_{\hat{\beta}}^{2}$, but it becomes biased when $\beta \neq 0$ due to the presence of the bias $\left(F^{T} h-b\right) \beta$, i.e. $\mathrm{E}\left(\hat{x}_{u_{B}}\right) \neq x_{u}$. To evaluate the stated bias, consider the least-squares inverse of $A_{u}$ as $A_{u}^{+}=\left(A_{u}^{T} Q_{y_{u} y_{u}}^{-1} A_{u}\right)^{-1} A_{u}^{T} Q_{y_{u} y_{u}}^{-1}$ with which the user computes the solutions $\hat{x}_{u_{A}}$ and $\hat{x}_{u_{B}}$ through (14) as

$\hat{x}_{u_{A}}=A_{u}^{+}\left(y_{u}+\hat{c}_{A}\right), \quad \hat{x}_{u_{B}}=A_{u}^{+}\left(y_{u}+\hat{c}_{B}\right)$

Taking the expectation of the above equations, together with (14) and $A_{u}^{+} A_{u}=I$, gives

$\mathrm{E}\left(\hat{x}_{u_{A}}\right)=x_{u}$, and $\mathrm{E}\left(\hat{x}_{u_{B}}\right)=x_{u}+A_{u}^{+}\left(F^{T} h-b\right) \beta$

Thus the user solution $\hat{x}_{u_{B}}$ is biased by $A_{u}^{+}\left(F^{T} h-b\right) \beta$ when $\beta \neq 0$. We are interested in a linear function of $x_{u}$, say $F_{u}^{T} x_{u}$. In an analogous way to (9), a link between the MSEs of the solutions $F_{u}^{T} \hat{x}_{u_{A}}$ and $F_{u}^{T} \hat{x}_{u_{B}}$ can be established. Here we assume that both $Q_{\hat{\hat{B}}_{\bar{B}} \hat{z}_{B}}$ and $Q_{\hat{\bar{z}}_{B} \hat{y}_{B}}$ from the network processing are delivered to the users and are thus known for calculation of the MSEs of $F_{u}^{T} \hat{x}_{u_{A}}$ and $F_{u}^{T} \hat{x}_{u_{B}}$.

Lemma 2 (MSES of $F_{u}^{T} \hat{x}_{u_{A}}$ and $F_{u}^{T} \hat{x}_{u_{B}}$ ). Consider the user observation Eq. (13) in which the observation vector $y_{u}$ is corrected to $\left(y_{u}+\hat{c}_{A}\right)$ and $\left(y_{u}+\hat{c}_{B}\right)$ using the networkderived corrections given in (11). The inverse variance matrix $Q_{y_{u} y_{u}}^{-1}$ is taken as weight matrix to compute the weighted leastsquares solutions $\hat{x}_{u_{A}}$ and $\hat{x}_{u_{B}}$ under Scenarios $A$ and $B$, respectively. The MSEs of $F_{u}^{T} \hat{x}_{u_{A}}$ and $F_{u}^{T} \hat{x}_{u_{B}}$ can then be given as

Scenario A : $\quad \operatorname{MSE}\left(F_{u}^{T} \hat{x}_{u_{A}}\right):=\mathrm{E}\left\|F_{u}^{T}\left(\hat{x}_{u_{A}}-x_{u}\right)\right\|^{2}=\operatorname{tr}\left(F_{u}^{T} Q_{\hat{x}_{u_{B}} \hat{x}_{u_{B}}} F_{u}\right)+\sigma_{\hat{\beta}}^{2} h_{u}^{T} h_{u}$ Scenario B : $\quad \operatorname{MSE}\left(F_{u}^{T} \hat{x}_{u_{B}}\right):=\mathrm{E}\left\|F_{u}^{T}\left(\hat{x}_{u_{B}}-x_{u}\right)\right\|^{2}=\operatorname{tr}\left(F_{u}^{T} Q_{\hat{x}_{u_{B}} \hat{x}_{u_{B}}} F_{u}\right)+\beta^{2} h_{u}^{T} h_{u}$

where 
$Q_{\hat{x}_{u_{B} \hat{x}_{u_{B}}}}=A_{u}^{+}\left(Q_{y_{u} y_{u}}+F^{T} Q_{\hat{\hat{B}}_{B} \hat{z}_{B}} F\right) A_{u}^{+T}, \quad$ and

$h_{u}=F_{u}^{T} A_{u}^{+}\left(F^{T} h-b\right)$

$$
\text { Matrix } A_{u}^{+}=\left(A_{u}^{T} Q_{y_{u} y_{u}}^{-1} A_{u}\right)^{-1} A_{u}^{T} Q_{y_{u} y_{u}}^{-1} \text { is a least-squares }
$$
inverse of $A_{u}$.

\section{Proof. see Appendix A.}

Compare (17) with (9). They are identical in structure. In both cases, the variance $\sigma_{\hat{\beta}}^{2}$ is accompanied by the multipliers $h^{T} h$ and $h_{u}^{T} h_{u}$. In contrast to $h^{T} h$ however, the term $h_{u}^{T} h_{u}$ does not necessarily tend to zero as $\epsilon \rightarrow 0$. This, namely, means that the MSE of the user solution $F_{u}^{T} \hat{x}_{u_{A}}$ can become unboundedly large when $\sigma_{\hat{\beta}}^{2} \rightarrow \infty$. This is in contrast to that of the network solution $\hat{z}_{A}$ in (9) which remains finite when $\sigma_{\hat{\beta}}^{2} \rightarrow \infty$. Such a difference is due to the 'dependency' of the network correction $c$, in (10), on the poorly estimable parameter $\beta$. The correction $c$ would, in turn, make the user parameters $F_{u}^{T} x_{u}$ dependent on $\beta$. Would the stated dependency be absent, i.e. $b=0$, the term $h_{u}$ would have reduced to $h_{u}=F_{u}^{T} A_{u}^{+} F^{T} h$ which tends to zero as $\sigma_{\hat{\beta}}^{2} \rightarrow \infty$. To gain a better insight into the dependency of $F_{u}^{T} x_{u}$ on $\beta$, suppose that the column vector $b$ lies in the range-space of the user design matrix $A_{u}$, i.e. $b=A_{u} \kappa$ for some $\kappa$. With $A_{u}^{+} A_{u}=I$, the column vector $h_{u}$ would then be specialized to

$h_{u}=F_{u}^{T}\left(A_{u}^{+} F^{T} h-\kappa\right)$

According to (19), in case of $h \approx 0$, both the MSEs in (17) are less influenced by $\sigma_{\hat{\beta}}^{2}$ and $\beta^{2}$ for linear functions of $x_{u}$ satisfying $F_{u}^{T} \kappa=0$. In that case, $F_{u}^{T} x_{u}$ is hardly dependent on $\beta$. When $F_{u}^{T} \kappa \neq 0$ however, the MSEs are more sensitive to $\sigma_{\hat{\beta}}^{2}$ and $\beta^{2}$. In the next subsection, we will exemplify such functions of the user parameters $x_{u}$.

\subsection{Network and user observation equations}

In this subsection the network model (5) and user model (13) are shown at work. In doing so, observation equations at the between-satellite single-differenced (SD) level are considered. As such SD network observation equations are 'rank-deficient' in the sense that not all parameters are unbiasedly estimable, the $\mathcal{S}$-system theory (Baarda, 1981; Teunissen, 1985a) is first employed to remove the underlying rank-deficiency. Instead of the original parameters, the resultant full-rank network model contains the socalled estimable parameters, distinguished from their origi-

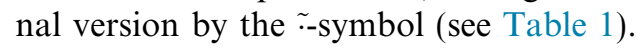

\subsubsection{Network-component}

Let the observed-minus-computed $(\mathrm{O}-\mathrm{C})$ terms of the carrier-phase and pseudo-range (code) observations of the network receiver $r(r=1, \ldots, n)$, tracked by satellite $s$ $(s=1, \ldots, m)$, be denoted by $\Delta \phi_{r, j}^{s}$ and $\Delta p_{r, j}^{s}$, respectively.
Table 1

Estimable GNSS parameters formed by a choice of $\mathcal{S}$-basis at the betweensatellite single-differenced level $(\cdot)^{1 s}=(\cdot)^{s}-(\cdot)^{1}$. The ZTDs $\tau_{r}$, ambiguities $a_{r, j}^{1 s}$ and satellite biases $\delta_{j}^{1 s} / d_{j}^{1 s}$ are assumed linked in time.

\begin{tabular}{ll}
\hline ZTDs & $\tilde{\tau}_{r}=\tau_{r} ; r=1,2, \ldots, n$ \\
Satellite clocks & $d \tilde{t}^{1 s}\left(t_{i}\right)=d^{1 s}\left(t_{i}\right)+d_{, I F}^{1 s}\left(t_{1}\right)$ \\
Ionospheric delays & $\tilde{l}_{r}^{1 s}\left(t_{i}\right)=t_{r}^{1 s}\left(t_{i}\right)-d_{, G F}^{1 s}\left(t_{1}\right) ; r=1,2, \ldots, n$ \\
Sat. phase biases & $\tilde{\delta}^{1 s}\left(t_{i}\right)=\delta_{j}^{1 s}\left(t_{i}\right)+\left(\mu_{j} d_{G F}^{1 s}\left(t_{1}\right)-d_{I F}^{1 s}\left(t_{1}\right)\right)-\lambda_{j} a_{1, j}^{1 s}$ \\
Sat. code biases & $\tilde{d}_{j}^{1 s}\left(t_{i}\right)=d_{j}^{1 s}\left(t_{i}\right)-\left(d_{, I F}^{1 s}\left(t_{1}\right)+\mu_{j} d_{, G F}^{s}\left(t_{1}\right)\right) ; j>2$ \\
& $\tilde{d}_{j}^{1 s}\left(t_{i>1}\right)=d_{, j}^{1 s}\left(t_{i}\right)-d_{, j}^{1 s}\left(t_{1}\right) ; j=1,2$ \\
Ambiguities & $\tilde{a}_{r, j}^{1 s}=a_{r, j}^{1 s}-a_{1, j}^{1 s} ; r \neq 1$ \\
$\mathcal{S}$-basis parameters & $a_{1, j}^{1 s}, d_{, 1}^{1 s}\left(t_{1}\right), d_{, 2}^{1 s}\left(t_{1}\right)$ \\
\hline
\end{tabular}

$(\cdot)_{, I F}=\frac{1}{\mu_{2}-\mu_{1}}\left[\mu_{2}(\cdot)_{, 1}-\mu_{1}(\cdot)_{, 2}\right] ;(\cdot)_{, G F}=\frac{1}{\mu_{2}-\mu_{1}}\left[(\cdot)_{, 2}-(\cdot)_{, 1}\right]$.

The subscript $j(j=1, \ldots, f)$ indicates the frequency on which the observations are collected. With the betweensatellite SD notation $(\cdot)^{1 s}=(\cdot)^{s}-(\cdot)^{1}$, a full-rank multifrequency GNSS network model reads (Odijk et al., 2016; Wang et al., 2017)

$$
\begin{aligned}
& \mathrm{E}\left(\Delta \phi_{r, j}^{1 s}\right)=g_{r}^{1 s} \tilde{\tau}_{r}-d \tilde{t}^{1 s}-\mu_{j} \tilde{l}_{r}^{1 s}-\tilde{\delta}_{j}^{1 s}+\lambda_{j} \tilde{a}_{r, j}^{1 s} \\
& \mathrm{E}\left(\Delta p_{r, j}^{1 s}\right)=g_{r}^{1 s} \tilde{\tau}_{r}-d \tilde{t}^{1 s}+\mu_{j} \tilde{l}_{r}^{1 s}-\tilde{d}_{, j}^{1 s}
\end{aligned}
$$

where $\tilde{\tau}_{r}$ denotes the estimable wet component of the ZTD accompanied by the Ifadis mapping function $g_{r}^{s}$ (Ifadis, 1986). The hydrostatic ZTD components are modelled as a priori values. The estimable satellite clock parameter is denoted by $d \tilde{t}^{l s}$. The estimable first-order ionospheric delay, experienced on the reference frequency $f_{1}$, is denoted by $\tilde{i}_{r}^{1 s}$. Thus the corresponding ionospheric coefficient is given as $\mu_{j}=f_{1}^{2} / f_{j}^{2}$. The frequency-dependent satellite phase and code biases are represented by $\tilde{\delta}_{, j}^{1 s}$ and $\tilde{d}_{, j}^{1 s}$, respectively. The estimable double-differenced (DD) ambiguities $\tilde{a}_{r, j}^{1 s}$ are linked to the observations through the wavelengths $\lambda_{j}$. All quantities are expressed in units of range, except the ambiguities $\tilde{a}_{r, j}^{1 s}$ which are given in cycles. The receiver-satellite geometry and biases like differential code biases (DCBs) and phase center variations (PCVs) are assumed to be removed by computing the $\mathrm{O}-\mathrm{C}$ terms. Here we remark that the high-order ionospheric delays can reach centimetres and are influenced by factors like station latitudes, time, solar cycles and relative geometry of the magnetic field (Hoque and Jakowski, 2007; Liu et al., 2016). In this contribution, they are assumed to be ignorable and are not taken into account in the observation model.

Small-scale networks. For the sake of presentation and simplicity, our focus is restricted to the single satellite pair $1-s$. The observation Eq. (20) then represent an example of the linear model (1) in which $\left[\Delta \phi_{r, j}^{1 s}, \Delta p_{r, j}^{1 s}\right]^{T}$ takes the role of $y$. Let us now assume that the network inter-station distances are short so that the receivers view satellite $s$ from almost the same direction angle. The tropospheric mapping functions can then be approximated by those of the reference receiver $r=1$, i.e. $g_{r}^{s} \approx g_{1}^{s}$. Thus $g_{r}^{1 s} \approx g_{1}^{1 s}, r=2$, $\ldots, n$. Under this assumption, the full-rank model (20) is shown to be near singular through (compare with (3)) 


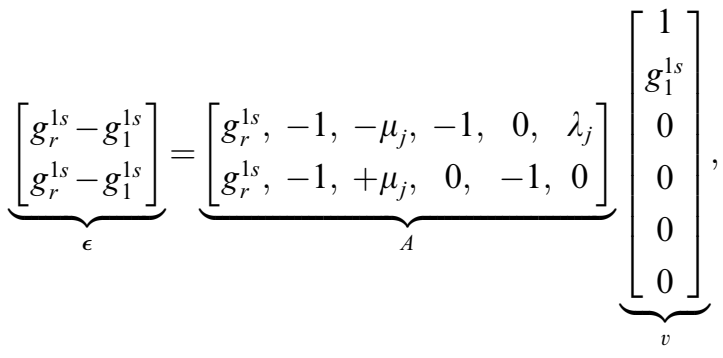

with $x=\left[\begin{array}{c}\tilde{\tau}_{r} \\ d \tilde{t}^{1 s} \\ \tilde{\imath}_{r}^{1 s} \\ \tilde{\delta}_{, j}^{1 s} \\ \tilde{d}_{, j}^{1 s} \\ \tilde{a}_{r, j}^{s}\end{array}\right]$

According to (21), there exists a near rank-deficiency between the estimable ZTDs $\tilde{\tau}_{r}(r=1, \ldots, n)$ and the satellite clocks $d \tilde{t}^{1 s}$ when $g_{r}^{1 s} \approx g_{1}^{1 s}$. Applying the transformation (4), the parameter vector $x$ can be expressed in terms of $\alpha$ and $\beta$-parameters as follows

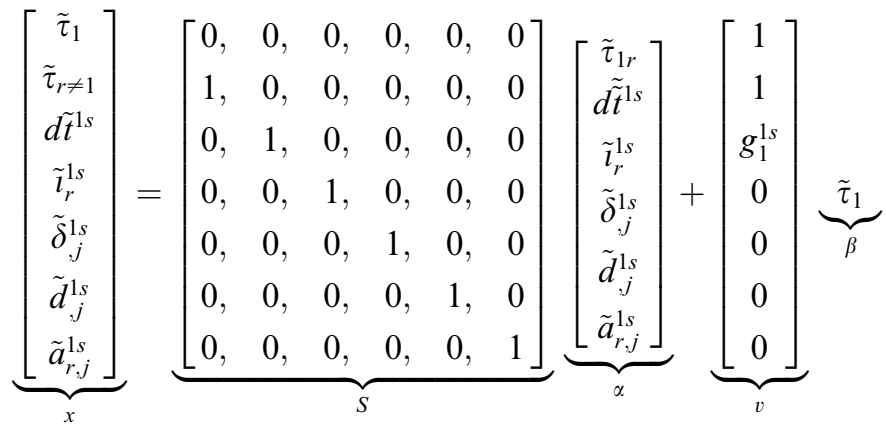

Thus the estimable ZTD of the reference receiver $r=1$, i.e. $\tilde{\tau}_{1}$, takes the role of the poorly estimable parameter $\beta$. The newly-defined parameters, given in vector $\alpha$, read

$\tilde{\tau}_{1 r}:=\tilde{\tau}_{r}-\tilde{\tau}_{1}, \quad \tilde{d \tilde{t}^{1 s}}:=d \tilde{t}^{1 s}-g_{1}^{1 s} \tilde{\tau}_{1}$

Substitution of (22) into (20) gives the counterpart of the network model (5) as

$$
\begin{aligned}
& \mathrm{E}\left(\Delta \phi_{r, j}^{1 s}\right)=g_{r}^{1 s} \tilde{\tau}_{1 r}-\tilde{d \tilde{t}^{1 s}}-\mu_{j} \tilde{l}_{r}^{1 s}-\tilde{\delta}_{, j}^{1 s}+\lambda_{j} \tilde{a}_{r, j}^{1 s}+g_{1 r}^{1 s} \tilde{\tau}_{1} \\
& \mathrm{E}\left(\Delta p_{r, j}^{1 s}\right)=g_{r}^{1 s} \tilde{\tau}_{1 r}-d \tilde{\tilde{t}}^{1 s}+\mu_{j} \tilde{l}_{r}^{1 s}-\tilde{d}_{, j}^{1 s}+g_{1 r}^{1 s} \tilde{\tau}_{1}
\end{aligned}
$$

where $g_{1 r}^{1 s}=g_{r}^{1 s}-g_{1}^{1 s}$. The above reparametrized network model clearly shows that the ZTD $\tilde{\tau}_{1}$ is weakly linked to the GNSS observations when $g_{1 r}^{1 s} \approx 0$. In that case, any linear combination of $\tilde{\tau}_{1}$ and $\alpha$ is poorly estimable. For instance, it follows from the first two rows of (22), i.e.

$\tilde{\tau}_{r}=\tilde{\tau}_{1 r}+\tilde{\tau}_{1}, \quad$ and $\quad d \tilde{t}^{1 s}=d \tilde{\tilde{t}}^{1 s}+g_{1}^{1 s} \tilde{\tau}_{1}$

that the absolute ZTDs $\tilde{\tau}_{r}(r=1, \ldots, n)$ and the estimable satellite clocks $d \tilde{t}^{1 s}(s=1, \ldots, m)$ are poorly estimable, since they are functions of $\tilde{\tau}_{1}$. This is however not the case with the relative ZTDs $\tilde{\tau}_{1 r}(r \neq 1)$ and $\tilde{\tilde{d}}^{1 s}$. Next to the other $\alpha$-parameters in (22), they form the parameter vector $z$ in (8), thereby having solutions with finite variances. The MSE expressions (9) do therefore hold for any linear functions of $z$ or equivalently $\alpha$ given in (22). Numerical evaluation will be presented in Section 3.1.

\subsubsection{Correction-component}

We now follow Scenarios A and B to obtain network solutions of the PPP-RTK correction $c$. The corrections include the estimable satellite clocks $d \tilde{t}^{1 s}$, phase/code biases $\tilde{\delta}_{, j}^{1 s} / \tilde{d}_{j}^{1 s}$ and the user slant ionospheric delays $\tilde{i}_{u}^{1 s}$. The correction $c$ reads then (cf. (10))

$$
c=\left[\begin{array}{c}
\tilde{\delta}_{j, j}^{1 s}+\mu_{j} \tilde{l}_{u}^{1 s}+d \tilde{t}^{1 s} \\
\tilde{d}_{, j}^{1 s}-\mu_{j} \tilde{l}_{u}^{1 s}+d \tilde{t}^{1 s}
\end{array}\right]=\underbrace{\left[\begin{array}{c}
\tilde{\delta}_{y}^{1 s}+\mu_{j} \tilde{l}_{u}^{1 s}+d \tilde{\tilde{t}^{1 s}} \\
\tilde{d}_{, j}^{1 s}-\mu_{j} \tilde{\imath}_{u}^{1 s}+d \tilde{\tilde{t}}^{1 s}
\end{array}\right]}_{F^{T}}+\underbrace{\left[\begin{array}{c}
g_{1}^{1 s} \\
g_{1}^{1 s}
\end{array}\right]}_{b} \underbrace{\tilde{\tau}_{1}}_{\beta}
$$

in which the estimable user ionospheric delay $\tilde{l}_{u}^{1 s}$ is assumed to follow as a weighted average of its network counterparts $\tilde{l}_{r}^{1 s}(r=1, \ldots, n)$. We note that the estimable ionospheric delays in Table 1 are contaminated by the hardware biases and to obtain precise ionospheric products for users, a dense network might be demanded. Instead of relying on ionosphere interpolation, Geng and Shi (2017) have also proposed a composite strategy to accelerate ambiguity resolution by simultaneously performing multi-GNSS PPP ambiguity resolution. In this study, since the GPS-only scenario was applied for the analysis, we assume the userspecific ionospheric delays are interpolated from those of the network stations and remark that the approach of ionosphere interpolation does not influence Lemma 2. As shown in (26), the correction $c$ depends on the poorly estimable parameter $\tilde{\tau}_{1}$ through the estimable satellite clocks $d \tilde{t}^{1 s}=d \tilde{\tilde{t}}^{1 s}+g_{1}^{1 s} \tilde{\tau}_{1}$. The network-derived solution $\hat{c}_{A}$ is obtained by keeping the ZTD $\tilde{\tau}_{1}$ as unknown, while $\hat{c}_{B}$ is obtained by constraining $\tilde{\tau}_{1}$ to zero.

\subsubsection{User-component}

The corrections $\hat{c}_{A}$ and $\hat{c}_{B}$ are to be separately applied to the user observation equations (compare with (13))

$$
\begin{aligned}
& \mathrm{E}\left(\left[\begin{array}{c}
\Delta \phi_{u, j}^{1 s} \\
\Delta p_{u, j}^{1 s}
\end{array}\right]\right)=\underbrace{\left[\begin{array}{c}
G_{u}^{1 s} \\
G_{u}^{1 s}
\end{array}\right] \Delta \tilde{x}_{u}+\left[\begin{array}{c}
\lambda_{j} \\
0
\end{array}\right] \tilde{a}_{u, j}^{1 s}+\left[\begin{array}{c}
g_{u}^{1 s} \\
g_{u}^{1 s}
\end{array}\right] \tilde{\tau}_{u}}_{A_{u} x_{u}} \\
& -\underbrace{\left(\left[\begin{array}{c}
\tilde{\delta}_{j}^{1 s}+\mu_{j} \tilde{l}_{u}^{1 s}+\tilde{d} \tilde{\tilde{t}}^{1 s} \\
\tilde{d}_{, j}^{1 s}-\mu_{j} \tilde{l}_{u}^{1 s}+d \tilde{\tilde{t}}^{1 s}
\end{array}\right]+\left[\begin{array}{c}
g_{1}^{1 s} \\
g_{1}^{1 s}
\end{array}\right] \tilde{\tau}_{1}\right)}_{c}
\end{aligned}
$$

where $\Delta \tilde{x}_{u}$ denotes the vector of unknown user coordinate increments, with the known design matrix $G_{u}^{1 s}$ containing satellite-to-receiver unit vectors. Thus the user parameter vector $x_{u}=\left[\Delta \tilde{x}_{u}^{T}, \tilde{a}_{u, j}^{1 s}, \tilde{\tau}_{u}\right]^{T}$ contains $\Delta \tilde{x}_{u}$, the estimable 
ambiguities $\tilde{a}_{u, j}^{1 s}$ and the user ZTD $\tilde{\tau}_{u}$. The MSE expressions (17) do therefore hold for any linear functions of $x_{u}$. To exemplify the condition (19), let us assume that the interstation distance between the user $u$ and the network receiver $r=1$ is short such that $g_{u}^{1 s} \approx g_{1}^{1 s}$. This yields (cf. (19))

$$
\underbrace{\left[\begin{array}{c}
g_{1}^{1 s} \\
g_{1}^{1 s}
\end{array}\right]}_{b} \approx \underbrace{\left[\begin{array}{ccc}
G_{u}^{1 s}, & \lambda_{j}, & g_{u}^{1 s} \\
G_{u}^{1 s}, & 0, & g_{u}^{1 s}
\end{array}\right]}_{A_{u}} \underbrace{\left[\begin{array}{l}
0 \\
0 \\
1
\end{array}\right]}_{\kappa}
$$

Thus the column vector $b$ almost lies in the range-space of the user design matrix $A_{u}$. According to the results presented in (19), the MSEs of the estimated position increments $\Delta \hat{\tilde{x}}_{u}$ and the ambiguities, which hold the condition $F_{u}^{T} \kappa=0$, are less sensitive to the large variance $\sigma_{\hat{\tau}_{1}}^{2}$ or the bias $\tilde{\tau}_{1}^{2}$ compared to those of $\hat{\tilde{\tau}}_{u}$, for which $F_{u}^{T}=[0,0,1]$ and $F_{u}^{T} \kappa=1$. We conclude this section by summarizing our findings as follows:

- Due to the near rank-deficiency between the ZTDs and the satellite clock parameters of small-scale networks, any linear functions of $\alpha$, given in (22), combined with the ZTD $\tilde{\tau}_{1}$ (i.e. $\beta$ ) are poorly estimable. Examples of which are the ZTDs $\tilde{\tau}_{r}=\tilde{\tau}_{1}+\tilde{\tau}_{1 r}(r=1, \ldots, n)$ and the estimable satellite clock parameters $d \tilde{t}^{1 s}=d \tilde{\tilde{t}}^{1 s}+g_{1}^{1 s} \tilde{\tau}_{1}$ $(s=2, \ldots, m)$.

- The $z$-parameters (8) do not depend on the poorly estimable parameter $\beta$ (e.g. $\tilde{\tau}_{1}$ ) in small-scale networks. The MSEs of their network solutions under Scenario A do therefore remain finite. Examples of which are the relative ZTDs $\tilde{\tau}_{1 r}$ and the estimable ambiguities $\tilde{a}_{r, j}^{1 s}$ (cf. (22)).

- In contrast to the network parameters $z$, the MSEs of user solutions $F_{u}^{T} \hat{x}_{u_{A}}$ can unboundedly get large when $\sigma_{\hat{\beta}}^{2} \rightarrow \infty$. For instance, the MSE of the estimated user ZTD $\hat{\tilde{\tau}}_{u_{A}}$ is largely affected by the variance $\sigma_{\hat{\beta}}^{2}$ (i.e. $\sigma_{\hat{\tau}_{1}}^{2}$ ). The MSEs of the estimated user positions and ambiguities however, are hardly affected by $\sigma_{\hat{\tau}_{1}}^{2}$.

- When the condition $\sigma_{\hat{\beta}}^{2}>\beta^{2}\left(\sigma_{\hat{\tau}_{1}}^{2}>\tilde{\tau}_{1}^{2}\right)$ holds, the MSE performance of both the network solutions $\hat{z}_{B}$ (or any linear function thereof) and the user solutions $F_{u}^{T} \hat{x}_{u_{B}}$ is better than that of their counterparts under Scenario A. One would then constrain the ZTD $\tilde{\tau}_{1}$ to zero to achieve better solutions in the MSE sense.

\section{Network and user solutions}

In this study, only formal analysis was performed and no real data was used. We simulated ground truth coordinates of network and user stations located in Australia, and the GPS final satellite orbits for the entire day of June 10, 2017 provided by the International GNSS Service (IGS,
Dow et al., 2009; Griffiths and Ray, 2009; IGS, 2017) were used for the study. The Curtin PPP-RTK Software was used to generate both the formal network and user solutions (Odijk et al., 2017; Wang et al., 2017) under GPS dual-frequency (L1 and L2) scenario with a sampling inter$\mathrm{val}$ of $30 \mathrm{~s}$. The processing was performed on a $2 \mathrm{~h}$ basis with the starting time of the processing at 0:00, 2:00, .., 22:00 in GPS Time (GPST). In case of the network processing, Kalman filtering was employed to compute 'multiepoch' network solutions. In this regard, the network ambiguities and ZTDs were assumed to be constant within the processing interval ( $2 \mathrm{~h}$ in this contribution), while the temporal behaviour of the satellite biases is modeled by a random walk process on undifferenced level with the process noise of $1 \mathrm{~cm} / \sqrt{\mathrm{sec}}$ (cf. Table 1) based on their stable but non-constant temporal behaviours (Wen et al., 2011). Both the network ambiguity-float and -fixed scenarios were considered. In case of the user processing, 'single-epoch' user solutions were obtained, i.e. the user parameters were assumed unlinked in time. The RMSEs of the network and user solutions are computed and compared under Scenarios A and B. To evaluate the RMSEs of the user solutions, the user ambiguities were assumed to be successfully resolved.

The carrier-phase and code data $\Delta \phi_{r, j}^{s}$ and $\Delta p_{r, j}^{s}$ are assumed uncorrelated, having the elevation-dependent variances (Dach et al., 2015)

$\sigma_{\phi_{r, j}^{s}}^{2}=\frac{\sigma_{\phi}^{2}}{\sin ^{2}\left(e_{r}^{s}\right)}, \quad \sigma_{p_{r, j}^{s}}^{2}=\frac{\sigma_{p}^{2}}{\sin ^{2}\left(e_{r}^{s}\right)}$

where $\sigma_{\phi}$ and $\sigma_{p}$ denote the zenith-referenced standard deviation of the carrier-phase and code observations, respectively. Here we set these standard-deviations to $\sigma_{\phi}=3 \mathrm{~mm}$ and $\sigma_{p}=25 \mathrm{~cm}$ (Odijk et al., 2014a). The satellite elevation angle from receiver $r$ to satellite $s$ is symbolized by $e_{r}^{s}$ with the elevation mask set to be 10 degrees. The average value

$\hat{\tilde{l}}_{u}^{1 s}=\frac{1}{n} \sum_{r=1}^{n} \hat{\tilde{l}}_{r}^{1 s}$

is used to provide the ionospheric correction $\hat{\tilde{l}}_{u}^{1 s}$ to the user, assuming that the user is located at the mean longitude, latitude and height of the network stations. As stated in Bevis et al. (1992) and Younes (2016), the wet ZTD could range from centimetres (or less) to $3.5 \mathrm{dm}$ depending on the humidity condition of the regions. As a result, different pre-defined $\tilde{\tau}_{1}$ varying from 0 to $3.5 \mathrm{dm}$ are used for the tests.

\subsection{Network solutions}

In this subsection, to illustrate the network part of the analytical derivations in Section 2 and to show examples of PPP-RTK network solutions, numerical results are shown and discussed with respect to the following aspects: 
- The behaviours of $\sigma_{\hat{\beta}}(6)$, or equivalently $\sigma_{\hat{\tau}_{1}}$ in small and large networks under Scenario A (cf. Fig. 2).

- The RMSEs of linear functions of $\hat{z}_{A}$, denoted as $F_{n}^{T} \hat{z}_{A}$, here as an example the $\operatorname{RMSE}\left(\hat{\tilde{\tau}}_{1 r}\right)$ under Scenario A, in small and large networks (cf. (9), Figs. 3 and 4).

- Change of the biases for $F_{n}^{T} \hat{z}_{B}$ with $\tilde{\tau}_{1}$, here as an example $\hat{\tilde{\tau}}_{1 r}$, in small and large networks; Comparison between $\operatorname{RMSE}\left(F_{n}^{T} \hat{z}_{A}\right)$ and $\operatorname{RMSE}\left(F_{n}^{T} \hat{z}_{B}\right)$, here as an example the comparison between $\operatorname{RMSE}\left(\hat{\tilde{\tau}}_{1 r}\right)$ under Scenarios A and B (cf. (9), Fig. 5 and Table 2).

- Change of the mean biases of ambiguities with $\tilde{\tau}_{1}$ under Scenario B in networks with different scales (cf. Fig. 6); Comparison of the network ambiguity success rates (ASRs) under Scenarios A and B for networks with different scales and different pre-defined $\tilde{\tau}_{1}$ (cf. Fig. 7).

As shown in Fig. 1, the simulated networks are located in west, north and east of Australia consisting of three stations 1, 2 and 3 each. The stations 1 (see the blue points in Fig. 1) are located at the longitudes of 116,133 and 147 degrees and the latitudes of $-30,-20$ and -30 degrees, respectively, and the stations 2 (see the red points in Fig. 1) are located in the east direction of the stations 1 with a 3-dimensional distance varying from 1 to $500 \mathrm{~km}$. The stations 3 (see the green points in Fig. 1) are located in north of the stations 1 and 2 with the same interstation distance between $1-3,2-3$ as between $1-2$. The heights (above ellipsoid) of all stations are $0 \mathrm{~m}$. The station 1 is used as the reference station for each network. We remark that only the satellites that are observed by all network stations are used for the processing.

As mentioned in Section 2, the variance of $\hat{\tilde{\tau}}_{1}$ is significantly influenced by the near-singularity in the network design matrix, when Scenario A is applied for small networks (6). With the increasing inter-station distance $d_{1 r}$ between stations 1 and $r$, the term $\epsilon$ that contains the between-receiver and between-satellite tropospheric mapping functions $g_{1 r}^{1 s}$ (21) also increases in magnitude. Fig. 2a shows the change of $g_{1 r}^{1 s}$ with respect to $d_{1 r}$ using the networks located in west of Australia at the first epoch of the test day for the reference satellite G27 and other commonly observed satellites. For different satellite pairs, linear change of $g_{1 r}^{1 s}$ with $d_{1 r}$ can be approximated in frame of this study, i.e., with $d_{1 r}$ varying from 1 to $500 \mathrm{~km}$, as:

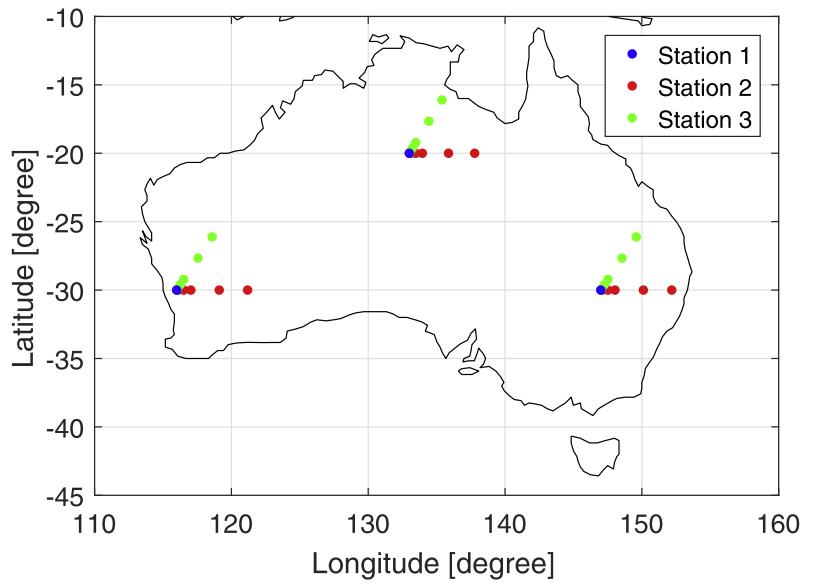

Fig. 1. Simulated networks located in west, north and east of Australia with inter-station distances varying from 1 to $500 \mathrm{~km}$.

$g_{1 r}^{1 s}=g_{r}^{1 s}-g_{1}^{1 s} \approx \dot{g}_{1}^{1 s} d_{1 r}$

where $\dot{g}_{1}^{1 s}$ represents the derivative of $g_{1}^{1 s}$ with respect to $d_{1 r}$. With the assumption that the satellites are distributed uniformly in the sky and are commonly observed by networks with different scales, the matrix $Q_{y y}$ in (6) is not assumed to have major changes as the network scale changes. The standard deviation $\sigma_{\hat{\beta}}$, or equivalently $\sigma_{\hat{\tau}_{1}}$, is almost inversely proportional to the inter-station distance $d_{1 r}$ (see (A)):

$\sigma_{\hat{\tau}_{1}} \approx \frac{1}{d_{1 r} \sqrt{\dot{\bar{\epsilon}}(0)^{T} Q_{y y}^{-1} \dot{\bar{\epsilon}}(0)}}$

where $\dot{\bar{\epsilon}}(0)$ is given in Appendix A. Fig. 2b shows the formal standard deviations of $\hat{\tilde{\tau}}_{1}$ using the network with $d_{1 r}$ of $1 \mathrm{~km}$ divided by a factor $q$ of 500 and those using the network with $d_{1 r}$ of $500 \mathrm{~km}$. The results of the first two hours of the day are processed under Scenario A with fixed and float ambiguities. Despite of the different numbers (see also Fig. 3c) and the non-uniform distribution of the satellites, the $\sigma_{\hat{\tau}_{1}}$ using the network with $d_{1 r}$ of $1 \mathrm{~km}$ (see the blue and red lines in Fig. 2b) are approximately 500 times larger than those using the network with $d_{1 r}$ of $500 \mathrm{~km}$ (see the green and magenta lines in Fig. 2b).

Based on the near-proportional relationship between $g_{1 r}^{1 s}$ and $d_{1 r}$ (31), without major changes in $Q_{\hat{z} B \hat{y}_{B}} Q_{y y}^{-1}$ when changing the network scale (A.5), the term

Table 2

The number of epochs $T\left(\Delta \operatorname{RMSE}\left(\hat{\tilde{\tau}}_{1 r}\right)<0\right)$ that is needed to turn $\Delta \operatorname{RMSE}\left(\hat{\tilde{\tau}}_{1 r}\right)$ between stations 1 and 2 from positive to negative values (34), and the number of epochs $T\left(\sigma_{\hat{\tau}_{1}}<\tilde{\tau}_{1}\right)$ that is needed to let $\sigma_{\hat{\tau}_{1}}$ smaller than $\tilde{\tau}_{1}$ (35). The networks located in west of Australia with inter-station distances $d_{1 r}$ of 50 and $500 \mathrm{~km}$ are processed for the first two hours of the test day. The values are given for different $\tilde{\tau}_{1}$ and networks with different inter-station distances $d_{1 r}$.

\begin{tabular}{|c|c|c|c|c|c|c|c|c|}
\hline \multirow[t]{3}{*}{$\tilde{\tau}_{1}, d_{1 r}$} & \multicolumn{4}{|c|}{$50 \mathrm{~km}$} & \multicolumn{4}{|c|}{$500 \mathrm{~km}$} \\
\hline & \multicolumn{2}{|c|}{$T\left(\Delta \mathrm{RMSE}\left(\hat{\tilde{\tau}}_{1 r}\right)<0\right)$} & \multicolumn{2}{|c|}{$T\left(\sigma_{\hat{\tilde{\tau}}_{1}}<\tilde{\tau}_{1}\right)$} & \multicolumn{2}{|c|}{$T\left(\Delta \mathrm{RMSE}\left(\hat{\tilde{\tau}}_{1 r}\right)<0\right)$} & \multicolumn{2}{|c|}{$T\left(\sigma_{\hat{\tau}_{1}}<\tilde{\tau}_{1}\right)$} \\
\hline & fixed & float & fixed & float & fixed & float & fixed & float \\
\hline $5 \mathrm{~cm}$ & 20 & 109 & 20 & 109 & 1 & 26 & 1 & 26 \\
\hline $1.5 \mathrm{dm}$ & 4 & 43 & 4 & 43 & 1 & 13 & 1 & 13 \\
\hline $3.5 \mathrm{dm}$ & 1 & 27 & 1 & 27 & 1 & 8 & 1 & 8 \\
\hline
\end{tabular}




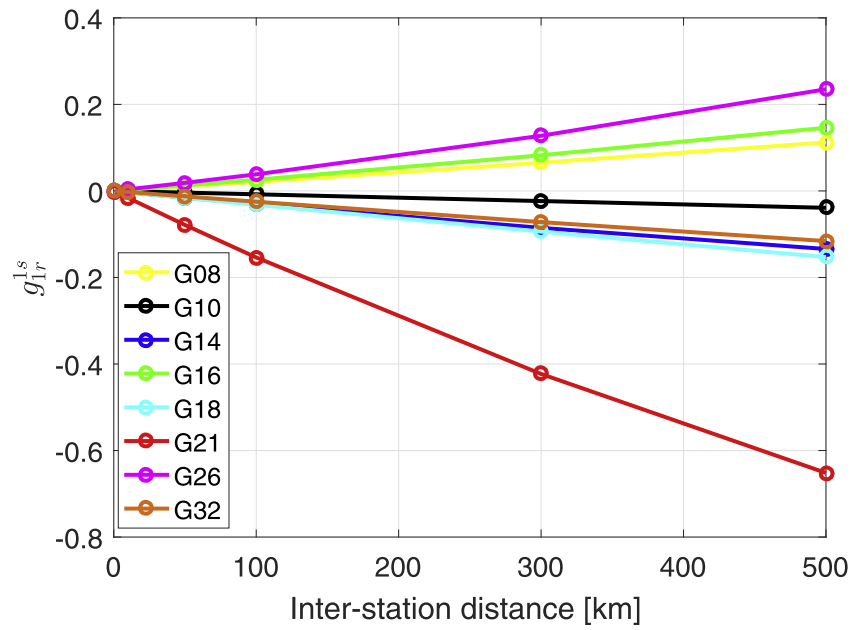

(a) $g_{1 r}^{1 s}$

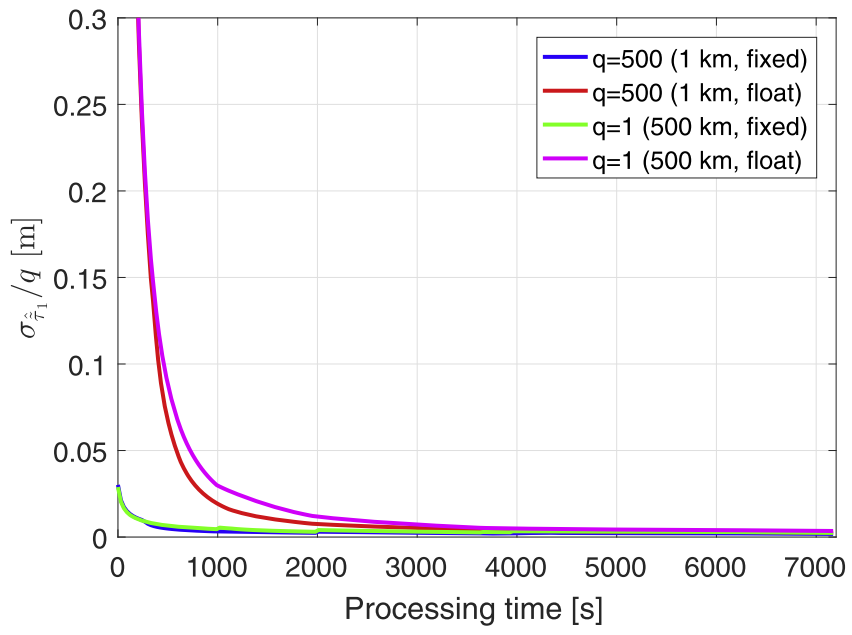

(b) $\sigma_{\hat{\tilde{\tau}}_{1}} / q$

Fig. 2. (a) The between-receiver and between-satellite tropospheric mapping functions $g_{1 r}^{1 s}$ at the first epoch of the test day using the networks located in west of Australia with different inter-station distances and (b) the formal standard deviations of $\hat{\tilde{\tau}}_{1}\left(\sigma_{\hat{\beta}}\right.$ in $\left.(6)\right)$ using the networks with inter-station distances of 1 and $500 \mathrm{~km}$ divided by factors $q$ of 500 and 1, respectively. The data is processed under Scenario A for the first two hours on June 10 , 2017 with fixed and float ambiguities for (b).

$\sqrt{\left(F_{n}^{T} h\right)^{T}\left(F_{n}^{T} h\right)}$ in (9) for any linear function of $z$, denoted as $F_{n}^{T} z$, or equivalently $F_{n}^{T} \alpha$, is near-proportional to the inter-station distance $d_{1 r}$ (A.5):

$$
\begin{gathered}
\sqrt{\left(F_{n}^{T} h\right)^{T}\left(F_{n}^{T} h\right)}=\sqrt{\left(F_{n}^{T} Q_{\hat{z}_{B} \hat{y}_{B}} Q_{y y}^{-1} \epsilon\right)^{T}\left(F_{n}^{T} Q_{\hat{z}_{B} \hat{y}_{B}} Q_{y y}^{-1} \boldsymbol{\epsilon}\right)} \\
\approx d_{1 r} \sqrt{\left(F_{n}^{T} Q_{\hat{z}_{B} \hat{y}_{B}} Q_{y y}^{-1} \dot{\boldsymbol{\epsilon}}\right)^{T}\left(F_{n}^{T} Q_{\hat{z}_{B} \hat{y}_{B}} Q_{y y}^{-1} \dot{\boldsymbol{\epsilon}}\right)}
\end{gathered}
$$

where $\dot{\boldsymbol{\epsilon}}$ contains the term $\dot{g}_{1}^{1 s}$ in (31). Taking the relative ZTDs between receivers 1 and 2 as an example, Fig. 3a shows the term $\sqrt{\left(F_{n}^{T} h\right)^{T}\left(F_{n}^{T} h\right)}$ using the same network with $d_{1 r}$ of $1 \mathrm{~km}$ (as in Fig. 2b) multiplied by a factor $q$ of 500 and that using the network with $d_{1 r}$ of $500 \mathrm{~km}$. The jumps are caused by changes in satellite geometry, which are shown in Fig. 3c. We see that despite of the different numbers and the non-uniform distribution of the satellites for both networks, the term $\sqrt{\left(F_{n}^{T} h\right)^{T}\left(F_{n}^{T} h\right)}$ of the small network with $d_{1 r}$ of $1 \mathrm{~km}$ is approximately 500 times smaller than that using the large network with $d_{1 r}$ of $500 \mathrm{~km}$. After multiplying them with $\sigma_{\hat{\tau}_{1}}(9)$, the term $d_{1 r}$ is eliminated based on (32) and (33). As shown in Fig. 3b, the product $\sigma_{\hat{\tau}_{1}} \sqrt{\left(F_{n}^{T} h\right)^{T}\left(F_{n}^{T} h\right)}$ is not that sensitive to the network scale anymore. Based on (9), since changing the network scales also does not lead to major changes in $\sigma_{\hat{\tau}_{1 r B}}$ (see Fig. 4a), the RMSEs of the relative ZTDs under Scenario $\mathrm{A}$ is at the same level for networks with $d_{1 r}$ of 1 and $500 \mathrm{~km}$ as shown in Fig. 4b. We remark that any linear combination of $\alpha$ and $\tilde{\tau}_{1}$ is poorly estimable for small networks under Scenario A, provided that the coefficients before $\tilde{\tau}_{1}$ are not zero. The parameters $\hat{\tilde{\tau}}_{r \neq 1}$ and $d \tilde{t}^{1 s}$ (22)

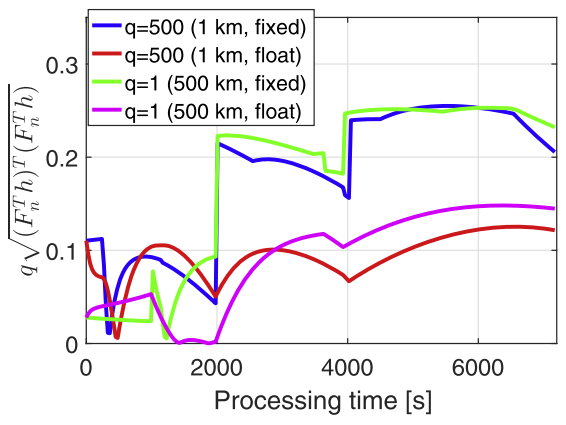

(a) $q \sqrt{\left(F_{n}^{T} h\right)^{T}\left(F_{n}^{T} h\right)}$

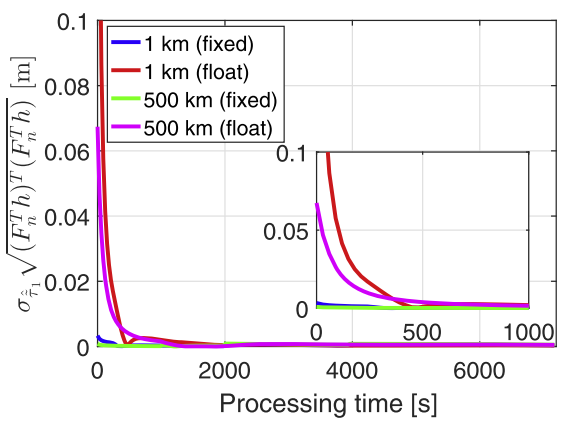

(b) $\sigma_{\hat{\tilde{\tau}}_{1}} \sqrt{\left(F_{n}^{T} h\right)^{T}\left(F_{n}^{T} h\right)}$

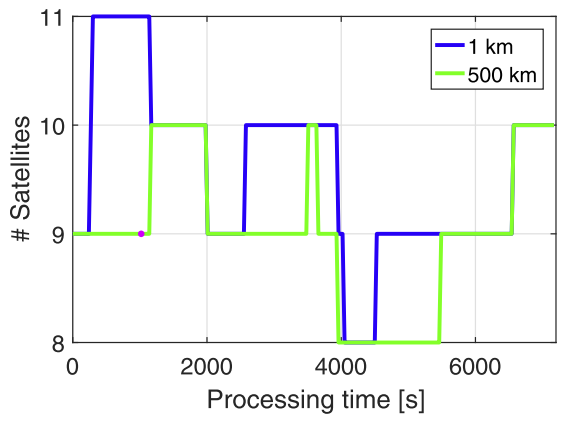

(c) No. Satellites

Fig. 3. (a) The term $\sqrt{\left(F_{n}^{T} h\right)^{T}\left(F_{n}^{T} h\right)}$ (33) for relative ZTDs between stations 1 and 2 using the networks located in west of Australia with inter-station

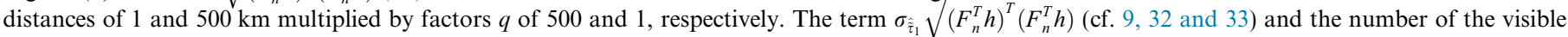
GPS satellites for both networks are plotted in (b) and (c). The data is processed for the first two hours on June 10, 2017. The lines in (b) are zoomed for the first 1000 epochs in the small window. The magenta point in (c) marks the time point with the same number of rising and setting satellites for the network with an inter-station distance of $500 \mathrm{~km}$. 


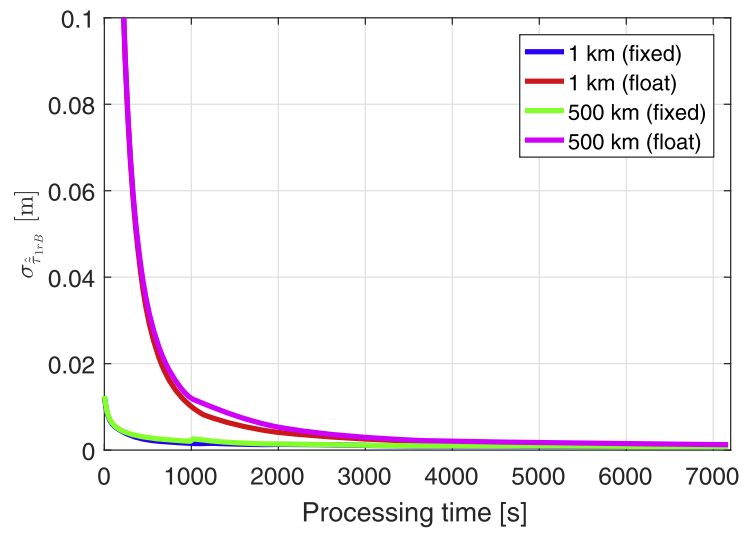

(a) $\sigma_{\hat{\tilde{\tau}}_{1 r B}}$

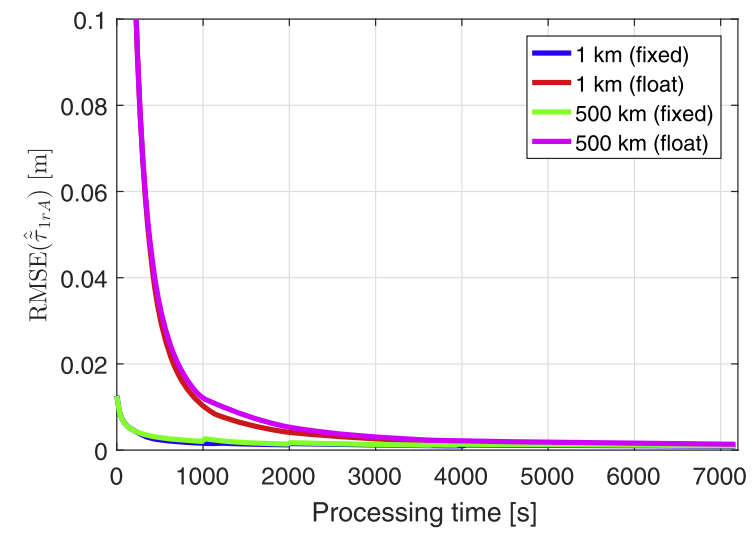

(b) $\operatorname{RMSE}\left(\hat{\tilde{\tau}}_{1 r A}\right)$

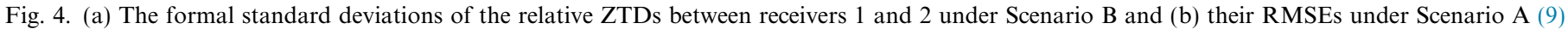

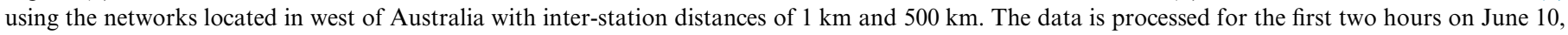
2017.

are two examples. For the small network with $d_{1 r}$ of $1 \mathrm{~km}$, their RMSEs under Scenario A reach hundreds to thousands of meters at the initialization phase in ambiguityfloat case as that for $\hat{\tilde{\tau}}_{1}$.

Under Scenario B, the parameter $\tilde{\tau}_{1}$ is constrained to be zero. This solves the near-singularity problem in the network design matrix for small networks under Scenario A. However, the term $g_{1 r}^{1 s} \tilde{\tau}_{1}$ (24) becomes mismodelled effect. Depending on the scale of the network, which leads to different sizes of the term $g_{1 r}^{1 s}$, and the humidity condition around the reference receiver, which leads to different values for $\tilde{\tau}_{1}$, employing Scenario B could bias the network and user solutions in a different manner. In Lemma 1, we note that the structure difference of MSEs for parameters $\alpha$ (and any linear function thereof) is only driven by $\sigma_{\hat{\tau}_{1}}$ and $\tilde{\tau}_{1}$. Different from $\sigma_{\hat{\tau}_{1}}$ that is near inversely proportional to $d_{1 r}(32), \tilde{\tau}_{1}$ is not related to the inter-station distance. As a result, for a certain $\tilde{\tau}_{1}$, the bias term $\tilde{\tau}_{1} \sqrt{\left(F_{n}^{T} h\right)^{T}\left(F_{n}^{T} h\right)}$ is near-proportional to $d_{1 r}$ as $\sqrt{\left(F_{n}^{T} h\right)^{T}\left(F_{n}^{T} h\right)}$ does (33). Fig. 5a and d show the bias term $\tilde{\tau}_{1} \sqrt{\left(F_{n}^{T} h\right)^{T}\left(F_{n}^{T} h\right)}(9)$, multiplied by a factor $q$, for the relative ZTDs between stations 1 and 2 during the first two hours of the test day with fixed and float ambiguities, respectively. The solid and dashed lines represent the cases using networks located in west of Australia with inter-station distances of 50 and $500 \mathrm{~km}$ and factors $q$ of 10 and 1 , respectively. We see that the bias term using the network with $d_{1 r}$ of $500 \mathrm{~km}$ is approximately 10 times larger than that using the network with $d_{1 r}$ of $50 \mathrm{~km}$. The bias increases with the increasing $\tilde{\tau}_{1}$ and has reached millimetres and centimetres for $d_{1 r}$ of 50 and $500 \mathrm{~km}$, respectively. For the small network with an inter-station distance of $1 \mathrm{~km}$ during these two hours, the bias term is within 2 sub-mm even with $\tilde{\tau}_{1}$ of $3.5 \mathrm{dm}$ due to the small $h$.
The biases directly influence the RMSEs of $\hat{\tilde{\tau}}_{1 r}$ under Scenario B (9). As shown in Fig. 5b and e, the RMSEs of $\hat{\tilde{\tau}}_{1 r B}$ using the network with $d_{1 r}$ of $500 \mathrm{~km}$ are approximately 10 times larger than those using the network with $d_{1 r}$ of $50 \mathrm{~km}$ after the initialization phase for large $\tilde{\tau}_{1}$, since the resulted biases during this time period are dominated in the RMSEs under Scenario B. During the initialization phase, or for small $\tilde{\tau}_{1}$ in ambiguity-float case (see the blue lines in Fig. 5e), the $\sigma_{\hat{\tau}_{1 r B}}$ (see Fig. 4a) have larger amplitudes than the bias terms. The RMSEs are thus dominated by $\sigma_{\hat{\tau}_{1 r i}}$, and the near-proportional relationship with $d_{1 r}$ does not hold anymore.

Using the RMSEs of $F_{n}^{T} \hat{\alpha}$ under Scenarios A and B, their differences are computed for networks with different scales and under different pre-defined $\tilde{\tau}_{1}$ as:

$\Delta \operatorname{RMSE}\left(F_{n}^{T} \hat{\alpha}\right)=\operatorname{RMSE}\left(F_{n}^{T} \hat{\alpha}_{A}\right)-\operatorname{RMSE}\left(F_{n}^{T} \hat{\alpha}_{B}\right)$

where $\hat{\alpha}_{A}$ and $\hat{\alpha}_{B}$ represents the estimated $\alpha$ under Scenarios $\mathrm{A}$ and $\mathrm{B}$, respectively. Using the same networks with $d_{1 r}$ of 50 and $500 \mathrm{~km}$ located in west of Australia, the RMSE differences (multiplied by the factors $q$ ) for the relative ZTDs between stations 1 and 2 are shown in Fig. $5 \mathrm{c}$ and $\mathrm{f}$ during the first two hours of the day for ambiguity-fixed and -float cases, respectively. Note that the near-proportional relationship between $\Delta \operatorname{RMSE}\left(\hat{\tilde{\tau}}_{1 r}\right)$ and $d_{1 r}$ only holds, when the RMSEs under Scenario A is much smaller than those under Scenario B with the bias terms dominated. For the network with $d_{1 r}$ of $500 \mathrm{~km}$ (see the dashed lines in Fig. 5c and $\mathrm{f}$ ), the degradation of the RMSEs resulted by the biases reach centimetres comparing the cases with large $\tilde{\tau}_{1}$ (see the green and red dashed lines in Fig. $5 \mathrm{c}$ and $\mathrm{f}$ ) and $\tilde{\tau}_{1}$ of zero (see the yellow dashed lines in Fig. $5 \mathrm{c}$ and $\mathrm{f}$ ). Based on Lemma 1 (9), the structure difference in the MSEs of $\hat{z}_{A}$ and $\hat{z}_{B}$ (and any linear function thereof) is only driven by the terms $\sigma_{\hat{\tau}_{1}}$ and $\tilde{\tau}_{1}$. We assume that the minimal 


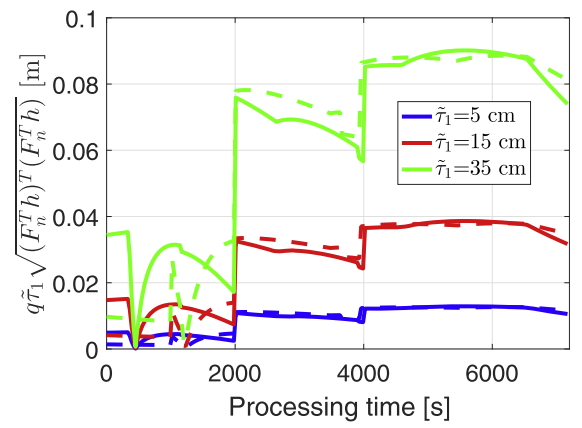

(a) $q \cdot \operatorname{Bias}($ fixed)

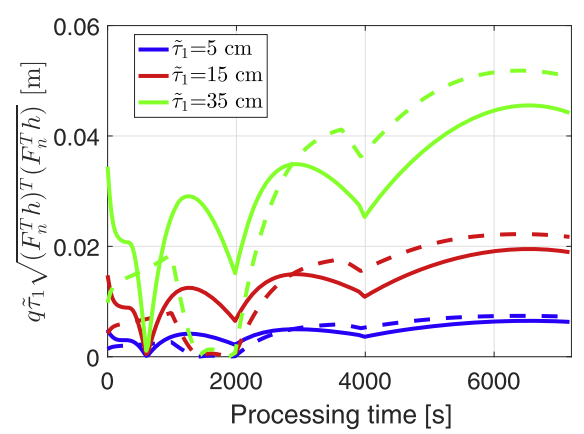

(d) $q$-Bias (float)

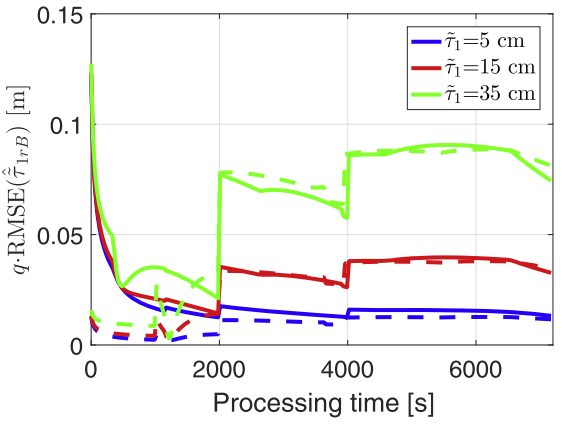

(b) $q \cdot \operatorname{RMSE}\left(\hat{\tilde{\tau}}_{1 r B}\right)$ (fixed)

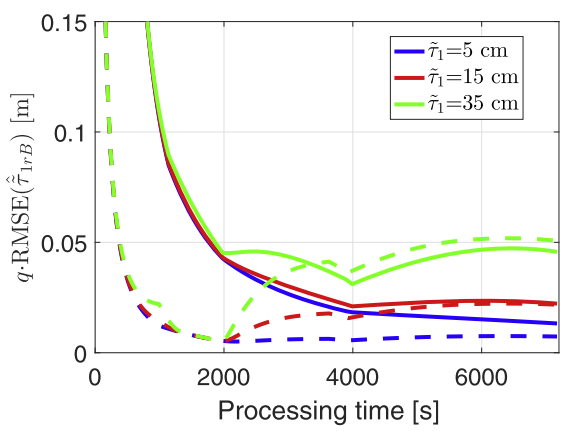

(e) $q \cdot \operatorname{RMSE}\left(\hat{\tilde{\tau}}_{1 r B}\right)$ (float)

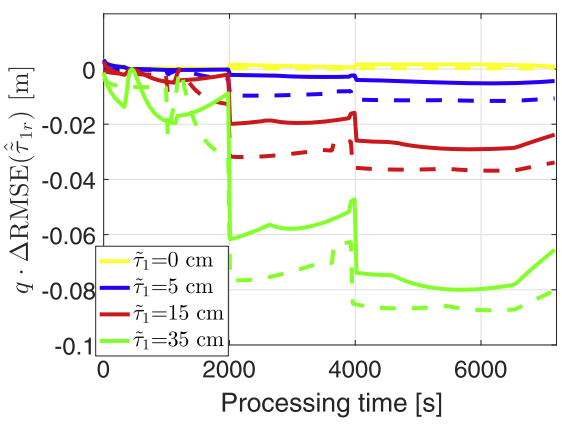

(c) $q \cdot \Delta \operatorname{RMSE}\left(\hat{\tilde{\tau}}_{1 r}\right)$ (fixed)

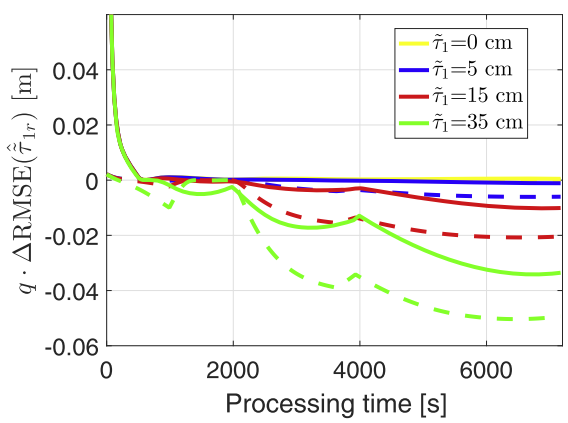

(f) $q \cdot \Delta \operatorname{RMSE}\left(\hat{\tilde{\tau}}_{1 r}\right)$ (float)

Fig. 5. The bias term $\tilde{\tau}_{1} \sqrt{\left(F_{n}^{T} h\right)^{T}\left(F_{n}^{T} h\right)}$ (9) multiplied by factor $q$ for relative ZTDs between stations 1 and 2 with (a) fixed and (d) float ambiguities, the corresponding RMSEs of $\hat{\tilde{\tau}}_{1 r}$ under Scenario B (9) and RMSE differences (34) multiplied by $q$ in (b, c) ambiguity-fixed and (e, f) -float cases. The solid and dashed lines represent the cases using the networks located in west of Australia with inter-station distances of 50 and $500 \mathrm{~km}$ with factors $q$ of 10 and 1 , respectively. The data is processed for the first two hours on June 10, 2017.

number of epochs that is needed to turn $\Delta \operatorname{RMSE}\left(\hat{\tilde{\tau}}_{1 r}\right)(34)$ from positive to negative values and that is needed to let $\sigma_{\hat{\tau}_{1}}$ become smaller than $\tilde{\tau}_{1}$ are defined as follows:

$$
\begin{aligned}
T\left(\Delta \operatorname{RMSE}\left(\hat{\tilde{\tau}}_{1 r}\right)<0\right) & =t_{\min }\left(\Delta \operatorname{RMSE}\left(\hat{\tilde{\tau}}_{1 r}\right)<0\right) \\
T\left(\sigma_{\hat{\tau}_{1}}<\tilde{\tau}_{1}\right) & =t_{\min }\left(\sigma_{\hat{\tau}_{1}}<\tilde{\tau}_{1}\right)
\end{aligned}
$$

where $t_{\min }(\cdot)$ is defined as the minimal number of epochs that is needed to fulfil the condition in $(\cdot)$. The terms $T\left(\Delta \operatorname{RMSE}\left(\hat{\tilde{\tau}}_{1 r}\right)<0\right)$ and $T\left(\sigma_{\hat{\tau}_{1}}<\tilde{\tau}_{1}\right)$ are listed in Table 2 for the two networks and different $\tilde{\tau}_{1}$ used in Fig. 5. Comparing the values for the networks with $d_{1 r}$ of 50 and 500 $\mathrm{km}$, we see that $\Delta \operatorname{RMSE}\left(\hat{\tilde{\tau}}_{1 r}\right)$ turns faster from positive to negative values in the larger network. For each predefined $\tilde{\tau}_{1}$ and network scale, the same number of epochs are required to turn $\triangle \mathrm{RMSE}\left(\hat{\tilde{\tau}}_{1 r}\right)$ from positive to negative and to let the $\sigma_{\hat{\tau}_{1}}$ become smaller than $\tilde{\tau}_{1}$ (cf. Lemma 1). If $\tilde{\tau}_{1}$ is set to zero, $\Delta \operatorname{RMSE}\left(\hat{\tilde{\tau}}_{1 r}\right)$ is above zero over the entire processing interval due to the fact that $\sigma_{\hat{\tau}_{1}}$ is larger than zero.

To compare the network ASRs under Scenario A without mismodelled effect and Scenario B with mismodelled effect, the integer least-squares ASRs are computed based on simulations ( $\mathrm{Li}$ et al., 2014). The deviations of the float ambiguities from their true values can be formulated for Scenarios A and B as:

$$
\begin{aligned}
\Delta \hat{\tilde{a}}_{A} & =\hat{\tilde{a}}_{A}-\tilde{a}=F_{\tilde{a}}^{T}\left(\hat{\alpha}_{A}-\alpha\right) \\
\Delta \hat{\tilde{a}}_{B} & =\hat{\tilde{a}}_{B}-\tilde{a}=F_{\tilde{a}}^{T}\left(\hat{\alpha}_{B}-\alpha\right)
\end{aligned}
$$

where $F_{\tilde{a}}^{T}$ selects all the estimable ambiguities from the vector $\alpha .10^{4}$ samples of the float ambiguity vector $\Delta \hat{\tilde{a}}_{A}$ are generated under Scenario $A$ using the variance matrix $F_{\tilde{a}}^{T} Q_{\hat{\alpha}_{A} \hat{\alpha}_{A}} F_{\tilde{a}}$ (A.8). Under Scenario B, $\Delta \hat{\tilde{a}}_{B}$ are generated with the help of the variance matrix $F_{\tilde{a}}^{T} Q_{\hat{\alpha}_{B} \hat{\alpha}_{B}} F_{\tilde{a}}$ (A.2) and the

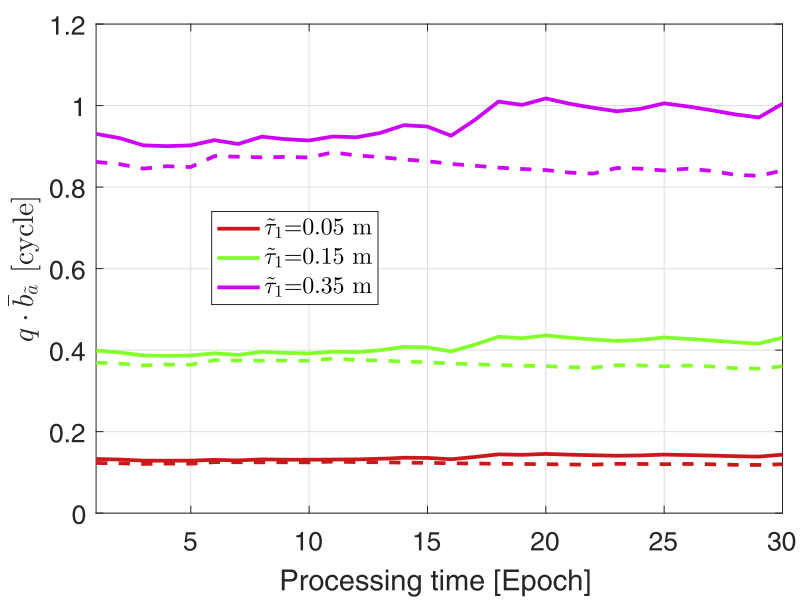

Fig. 6. The mean biases $\bar{b}_{\tilde{a}}$ of ambiguities under Scenario B (37) for networks with inter-station distances of $50 \mathrm{~km}$ (solid lines) and $500 \mathrm{~km}$ (dashed lines) that are multiplied by factors $q$ of 10 and 1, respectively. 
term $F_{\tilde{a}}^{T} h \tilde{\tau}_{1}$ (A.9) using a pre-defined $\tilde{\tau}_{1}$. In Fig. 6, the mean biases for ambiguities $\bar{b}_{\tilde{a}}$ under Scenario B using networks with inter-station distances of 50 and $500 \mathrm{~km}$ are multiplied by factors $q$ of 10 and 1, respectively, and are plotted for the first 30 epochs of the processing. Networks located in different areas of Australia and all processing intervals are used to compute the mean ambiguity biases $\bar{b}_{\tilde{a}}$ as follows:

$\bar{b}_{\tilde{a}}=\frac{\sum_{k=1}^{K} \sum_{h=1}^{H} b_{\tilde{a}}(k, h)}{K \cdot H}$

where $K$ and $H$ represent the number of the areas and the number of the processing time intervals, respectively. The term $b_{\tilde{a}}(k, h)$ represents the mean absolute biases of ambiguities under Scenario B for the network located in area $k$ during the processing interval $h$ with $b_{\tilde{a}}$ defined as (cf. (9)):

$b_{\tilde{a}}=\tilde{\tau}_{1} \frac{\sum\left|F_{\tilde{a}}^{T} h\right|}{l_{\tilde{a}}}$

where $\sum\left|F_{\tilde{a}}^{T} h\right|$ represents the sum of all the elements $\left|F_{\tilde{a}}^{T} h\right|$, and $l_{\tilde{a}}$ represents the number of the estimable ambiguities at the corresponding epoch.

As the bias terms for the relative ZTDs shown in Fig. 5a and d, from Fig. 6 we see that the mean ambiguity biases using networks with $d_{1 r}$ of $500 \mathrm{~km}$ are approximately 10 times larger than those using networks with $50 \mathrm{~km}$. For large networks with $d_{1 r}$ of $500 \mathrm{~km}$ (see the dashed lines in Fig. 6), the mean biases of the ambiguities could reach deci-cycles to cycles. This could significantly influence the float ambiguities and the ASRs under Scenario B. The float ambiguities are resolved with the LAMBDA method (Teunissen, 1993, 1995) at each epoch, and the ASRs are calculated for both scenarios with:

$P_{s}=\frac{P_{0}}{P}$

where $P_{0}$ represents the number of the samples with all the resolved ambiguities (after decorrelation) equal to zero, and $P$ denotes the total number of samples. The mean ASRs $\bar{P}_{s}$ are calculated using the networks located in different areas and during all the processing intervals as:

$\bar{P}_{s}=\frac{\sum_{k=1}^{K} \sum_{h=1}^{H} P_{s}(k, h)}{K \cdot H}$

where $P_{s}(k, h)$ represents the ASRs for the network located in area $k$ during the processing interval $h$. Fig. 7 shows the $\bar{P}_{s}$ (40) during the first 30 epochs of the processing for networks with inter-station distances of $50 \mathrm{~km}$ and $500 \mathrm{~km}$. With $\tilde{\tau}_{1}$ set to $0 \mathrm{~m}$, the $\bar{P}_{s}$ under Scenario B (see the blue lines in Fig. 7) are higher than those under Scenario A (see the yellow lines in Fig. 7), especially at the initialization phase. However, with increasing $\tilde{\tau}_{1}$, the biases could strongly degrade the ASRs under Scenario B. Using Scenario $\mathrm{B}$ for networks with an inter-station distance of 50 $\mathrm{km}$ and $\tilde{\tau}_{1}$ of $1.5 \mathrm{dm}$ (see the green line in Fig. 7a), the mean ASR turns to be lower than that under Scenario A after 7 epochs. For large networks with an inter-station distance of $500 \mathrm{~km}$ and a pre-defined $\tilde{\tau}_{1}$ of $1.5 \mathrm{dm}$, as shown by the green line in Fig. $7 \mathrm{~b}$, the mean ASRs are almost zero under Scenario B. We remark that the comparison of the ASRs are different from the comparison of the RMSEs of the ambiguities, since the biases do not only influence the diagonal elements, but also the other elements in the matrix $\mathrm{E}\left(\left(\hat{\tilde{a}}_{B}-\tilde{a}\right)\left(\hat{\tilde{a}}_{B}-\tilde{a}\right)^{T}\right)$. This would also affect the ASRs under Scenario B.

\subsection{User solutions}

The RMSEs of the user parameter solutions are also computed and compared using network corrections under Scenarios A and B with different pre-defined values of $\tilde{\tau}_{1}$. As mentioned at the beginning of Section 3, the users are assumed to be located at the mean longitude, latitude and height of the network stations. The network corrections are provided to the user at each epoch from the start of the network processing, and the single-epoch user solutions are evaluated with the ambiguities fixed. To compare the RMSEs of the estimated user coordinates under both scenarios, their differences are computed for networks with different scales and under different pre-defined $\tilde{\tau}_{1}$ :

$\Delta \operatorname{RMSE}\left(\Delta \hat{\tilde{x}}_{u}\right)=\operatorname{RMSE}\left(\Delta \hat{\tilde{x}}_{u A}\right)-\operatorname{RMSE}\left(\Delta \hat{\tilde{x}}_{u B}\right)$

where $\Delta \hat{\tilde{x}}_{u A}$ and $\Delta \hat{\tilde{x}}_{u B}$ represent the estimated user coordinate increments under Scenarios A and B, respectively. The comparison of the RMSEs of the estimated user coordinates is performed using all tested networks in west, north and east of Australia and all the processing intervals during the test day. The mean RMSE differences are computed for each pre-defined $\tilde{\tau}_{1}$ and each inter-station distance as follows:

$\Delta \operatorname{RMSE}\left(\Delta \hat{\tilde{\tilde{x}}}_{u}\right)=\frac{\sum_{k=1}^{K} \sum_{h=1}^{H} \Delta \operatorname{RMSE}\left(\Delta \hat{\tilde{x}}_{u}\right)(k, h)}{K \cdot H}$

where $\Delta \operatorname{RMSE}\left(\Delta \hat{\tilde{x}}_{u}\right)(k, h)$ represents the RMSE differences of the estimated user coordinates for network located in area $k$ during the test time interval $h$. The mean RMSE differences in the estimated user coordinates are shown in Fig. 8 for pre-defined $\tilde{\tau}_{1}$ varying from $5 \mathrm{~mm}$ to $3.5 \mathrm{dm}$ and networks with inter-station distances of 300 and 500 $\mathrm{km}$. The results are shown for both the network ambiguity-fixed (top-panel) and -float (bottom-panel) cases. The user ambiguities are assumed to be resolved. In case of the ambiguity-fixed network corrections, we see that the $\Delta \operatorname{RMSE}\left(\Delta \hat{\tilde{\tilde{x}}}_{u}\right)(42)$ are either slightly above zero for small $\tilde{\tau}_{1}$ with the amplitude within 0.2 sub-mm (see the blue lines in Fig. 8a and b), or below zero with their absolute values increasing with the increasing $\tilde{\tau}_{1}$ and network scales due to the enlarged biases under Scenario B. For smaller networks with inter-station distances within 100 $\mathrm{km}$, the absolute values of $\Delta \operatorname{RMSE}\left(\Delta \hat{\tilde{\tilde{x}}}_{u}\right)$ with even the largest $\tilde{\tau}_{1}$ in our test, i.e., $3.5 \mathrm{dm}$, are within 1 sub-mm. This 


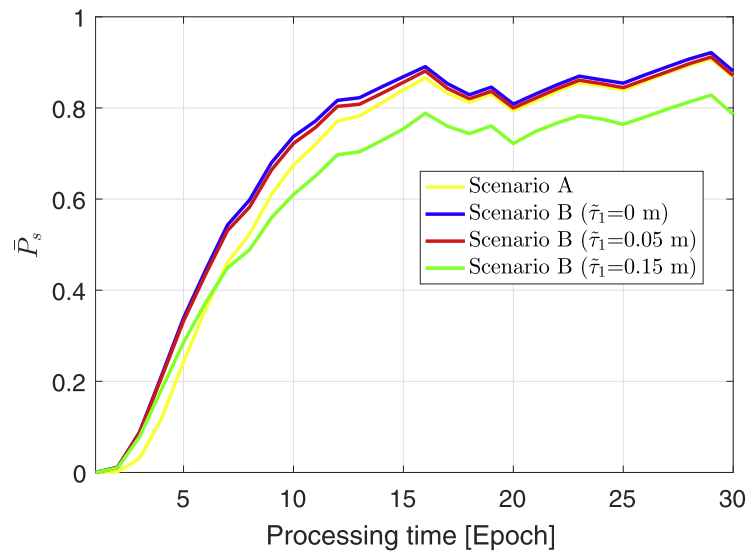

(a) $50 \mathrm{~km}$

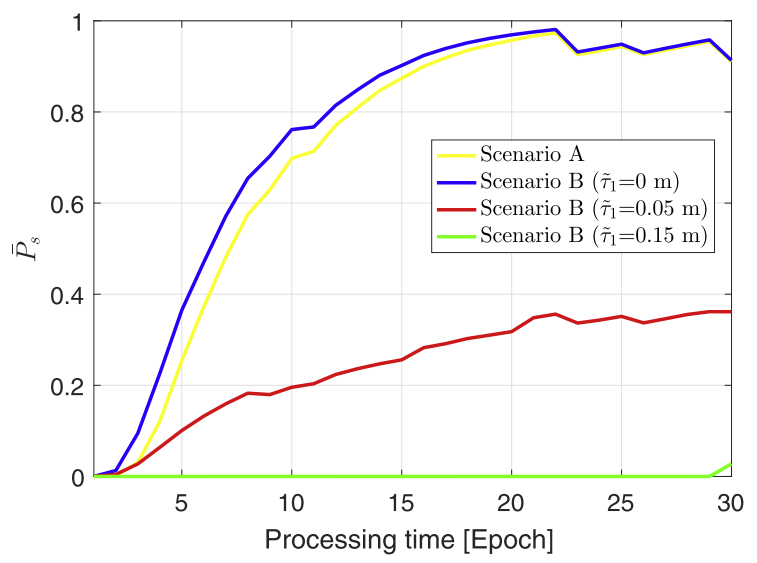

(b) $500 \mathrm{~km}$

Fig. 7. Simulated mean ambiguity success rates $\bar{P}_{s}(40)$ under Scenarios A and B for networks with inter-station distances of (a) $50 \mathrm{~km}$ and (b) $500 \mathrm{~km}$.

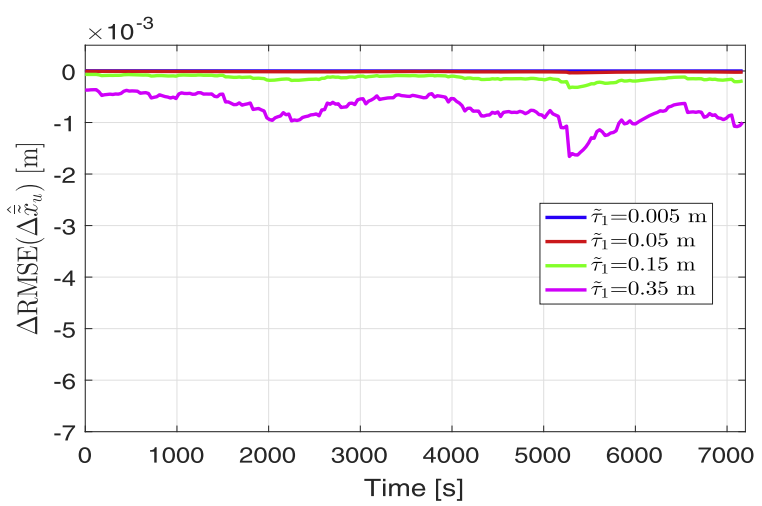

(a) $300 \mathrm{~km}$ (net. amb. fixed)

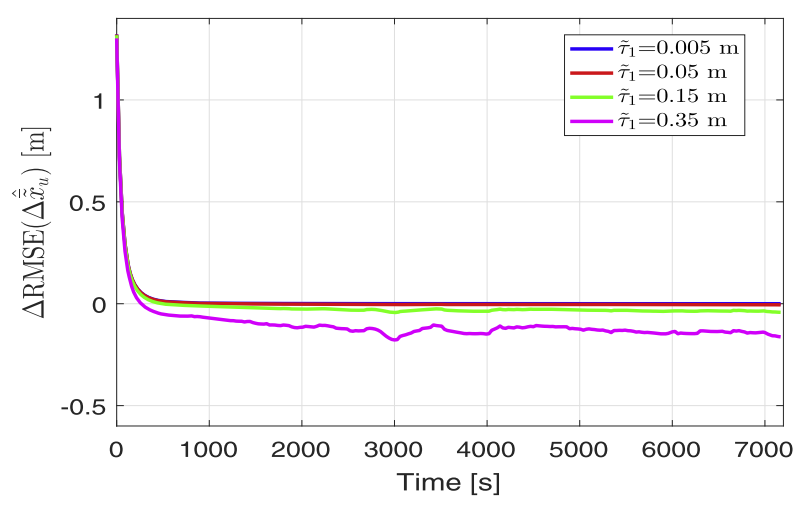

(c) $300 \mathrm{~km}$ (net. amb. float)

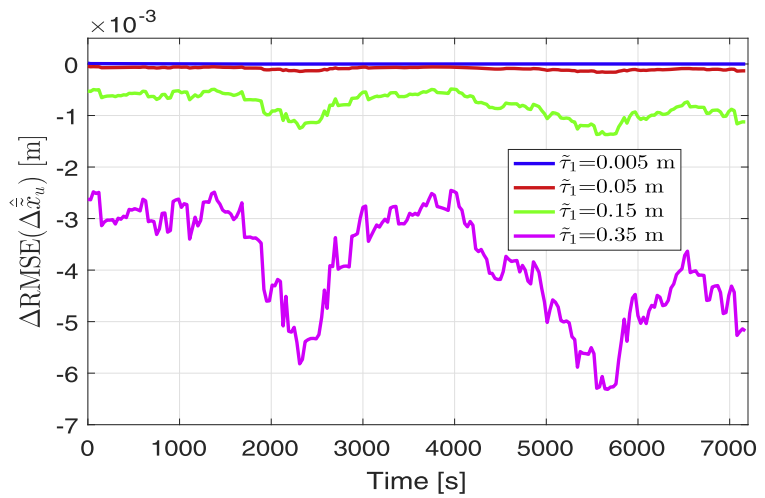

(b) $500 \mathrm{~km}$ (net. amb. fixed)

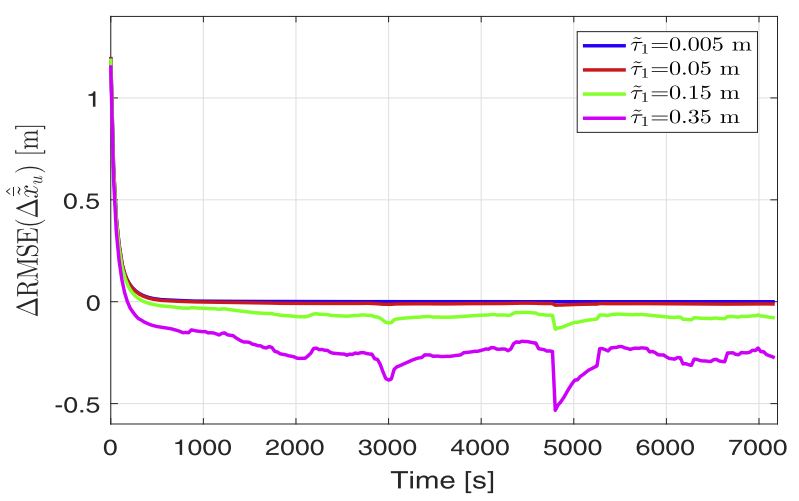

(d) $500 \mathrm{~km}$ (net. amb. float)

Fig. 8. Mean RMSE differences (42) of the estimated user coordinates for networks with inter-station distances of $300 \mathrm{~km}$ (left) and $500 \mathrm{~km}$ (right) where ambiguity-fixed (top) and -float (bottom) network-corrections are applied. The user ambiguities are assumed to be resolved. We note that in (c) the blue line is almost overwritten by the red line. (For interpretation of the references to color in this figure legend, the reader is referred to the web version of this article.)

indicates that in ambiguity-fixed cases at both the network and the user side, for networks with an inter-station distance shorter than $100 \mathrm{~km}$, the choices of the ZTD estimation strategy is not crucial for user positioning results. For networks with inter-station distances larger than $300 \mathrm{~km}$ in very humid regions (for the reference receiver) with $\tilde{\tau}_{1}$ of
$3.5 \mathrm{dm}$, as shown by the magenta lines in Fig. $8 \mathrm{a}$ and b, the RMSEs under Scenario B are higher than those under Scenario A with differences within millimetres.

In case of the ambiguity-float network corrections, when networks with inter-station distances within $100 \mathrm{~km}$ are considered, the $\Delta \operatorname{RMSE}\left(\Delta \hat{\tilde{\tilde{x}}}_{u}\right)$ under different $\tilde{\tau}_{1}$ are almost 
overwritten by each other and are thus not shown in the figure. At the initialization phase of the network filtering, the mean RMSEs of the user coordinates under Scenario A are higher than those under Scenario B with differences up to meters. The mean RMSE differences decrease rapidly from positive to negative values for large $\tilde{\tau}_{1}$ (see the magenta lines in Fig. $8 \mathrm{c}$ and d), while with $\tilde{\tau}_{1}$ of $5 \mathrm{~mm}$ for networks with an inter-station distance of $300 \mathrm{~km}$, the $\Delta \operatorname{RMSE}\left(\Delta \hat{\tilde{\tilde{x}}}_{u}\right)$ remain positive during the entire $2 \mathrm{~h}$ processing interval (see the blue line in Fig. 8c). If the reference receiver is located in humid regions with $\tilde{\tau}_{1}$ of $1.5 \mathrm{dm}$ (see the green lines in Fig. 8c and d), for networks with interstation distances of 100,300 and $500 \mathrm{~km}$, the RMSEs under Scenario B after the initialization phase could be higher than those under Scenario A with differences up to millimetres, centimetres and decimetres, respectively.

To search for the minimal number of the epochs that is needed to turn the mean RMSE differences $\triangle \operatorname{RMSE}\left(\Delta \hat{\tilde{\tilde{x}}}_{u}\right)$ from positive to negative values, $T_{0}$ is defined as follows:

$T_{0}=t_{\min }\left(\Delta \operatorname{RMSE}\left(\Delta \hat{\tilde{\tilde{x}}}_{u}\right)<0\right)$

In case that $\Delta \operatorname{RMSE}\left(\Delta \hat{\tilde{\tilde{x}}}_{u}\right)$ are positive during the entire processing time interval, i.e., 240 epochs, $T_{0}$ is set to be 241 epochs. Using ambiguity-float network corrections, Fig. 9 shows the term $T_{0}$ (43) for different pre-defined $\tilde{\tau}_{1}$ and networks with different scales. We see that $T_{0}$ decreases with the increasing inter-station distance and $\tilde{\tau}_{1}$. As shown by the blue line in Fig. 9, if the reference receiver is located in arid region with $\tilde{\tau}_{1}$ of $5 \mathrm{~mm}$, using Scenario B always generates smaller mean RMSEs during the $2 \mathrm{~h}$ processing time for an inter-station distance up to $300 \mathrm{~km}$. In regions with $\tilde{\tau}_{1}$ of $5 \mathrm{~cm}, 1.5 \mathrm{dm}$ and $3.5 \mathrm{dm}$, for networks with an

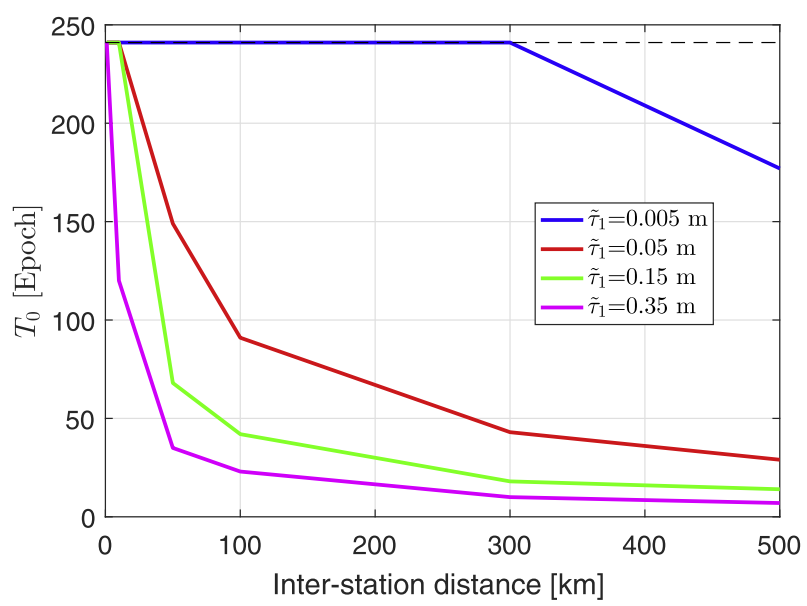

Fig. 9. Minimal number of epochs that is needed to turn the mean RMSE differences of the estimated user coordinates $\Delta \operatorname{RMSE}\left(\Delta \hat{\tilde{\tilde{x}}}_{u}\right)$ (43) from positive to negative values. The ambiguity-float network corrections are used for the processing with user ambiguities assumed to be fixed. The $T_{0}$ is set to be 241 epochs (see the black dashed line), if it exceeds the $2 \mathrm{~h}$ processing interval of 240 epochs. inter-station distance of $100 \mathrm{~km}$, it takes around 90, 40 and 20 epochs to switch from Scenario B to Scenario A, respectively.

\section{Conclusions}

The wet component of the zenith tropospheric delay (ZTD) is one of the unknowns in PPP-RTK processing. In small networks, the tropospheric mapping functions of different receivers to the same satellite are almost identical to each other. In the design matrix, the columns for the estimable ZTDs and satellite clocks are thus almost linearly dependent. To solve this problem, in small networks, the wet ZTD components are often estimated relatively to that of the reference receiver with the wet ZTD component of the reference receiver constrained. However, as the network scale and the wet ZTD component of the reference receiver increase, the mismodelled effect in case of relative ZTD estimation is enlarged, which could bias both the network and the user solutions. This contribution aimed to study and compare the accuracies of the network and the user solutions under.

- Scenario A: Absolute ZTD estimation at the network processing

- Scenario B: Relative ZTD estimation at the network processing

The MSE and its square root RMSE, which consider both the influences of the noise and biases, are used to evaluate the accuracies of the network and user solutions.

It was found that for small networks under Scenarios A, the near-singularity in the network design matrix mainly influences the MSEs of the estimated wet ZTD component of the network and the user stations, as well as the original estimable satellite clocks. The estimates of the relative ZTDs, the ambiguities, the user coordinates and other estimable parameters are less sensitive to the nearsingularity in the network design matrix. For these parameters, the difference in the MSE structures under Scenarios $\mathrm{A}$ and $\mathrm{B}$ is only driven by the square of the wet ZTD component of the reference receiver and the variance of its solution.

These conclusions were illustrated by simulation studies using GPS dual-frequency $30 \mathrm{~s}$ data for networks consisting of 3 stations. In addition to that, simulations were also performed to compute ASRs under both scenarios considering also the mismodelled effects. It was found that if the reference receiver is located in humid regions, using Scenario B for large networks could significantly degrade the ASRs due to the large biases. Using ambiguity-fixed network corrections, the mean RMSE differences of the estimated user coordinates between Scenarios A and B are within 1 sub-mm for networks with inter-station distances within $100 \mathrm{~km}$, even when the reference receiver is located in very humid regions. For larger networks with interstation distances of 300 and $500 \mathrm{~km}$ with an extreme $\tilde{\tau}_{1}$ 
of $3.5 \mathrm{dm}$, the mean RMSEs of the user coordinate estimates under Scenario B are higher than those under Scenario A with differences within millimetres. Using ambiguity-float network corrections, the RMSEs under Scenario A are larger than those under Scenario B by meters at the initialization phase. For networks with an inter-station distance of $100 \mathrm{~km}$, it takes around 90, 40 and 20 epochs to switch the mean RMSE differences between Scenarios A and B from positive to negative values for $\tilde{\tau}_{1}$ of $5 \mathrm{~cm}, 1.5 \mathrm{dm}$ and $3.5 \mathrm{dm}$, respectively. After the initialization phase, for networks with inter-station distances of 100,300 and $500 \mathrm{~km}$ and with $\tilde{\tau}_{1}$ of $1.5 \mathrm{dm}$, the RMSEs of the user coordinate estimates under Scenario B could be higher than those under Scenario A with differences up to millimetres, centimetres and decimetres, respectively.

\section{Acknowledgments}

We would like to thank the International GNSS Service (IGS) for providing the orbit products. The orbit products were obtained through the online archives of the Crustal Dynamics Data Information System (CDDIS), NASA Goddard Space Flight Center, Greenbelt, MD, USA. $\mathrm{ftp} / / /$ cddis.gsfc.nasa.gov/pub/gnss/products/. Special thanks also go to our colleagues in the GNSS Research Centre, Curtin University, for the development of the Curtin PPP-RTK Software. P.J.G. Teunissen is recipient of an Australian Research Council (ARC) Federation Fellowship (project number FF0883188).

\section{Appendix A.}

Proof of Lemma 1. We first follow Scenario $B$ and constrain $\beta$ to zero. Substitution into (5) gives the inconsistent linear system

$y \approx A S \alpha$

With the weight matrix $Q_{y y}^{-1}$, the weighted least-squares solution $\hat{\alpha}_{B}$ follows from (Teunissen, 2000)

$\hat{\alpha}_{B}=\left(S^{T} N S\right)^{-1} S^{T} A^{T} Q_{y y}^{-1} y, \quad$ with $\quad Q_{\hat{\alpha}_{B} \hat{\alpha}_{B}}=\left(S^{T} N S\right)^{-1}$

From (8), the least-squares solution $\hat{z}_{B}$ follows as

$$
\begin{aligned}
\hat{z}_{B} & =L^{T} S\left(S^{T} N S\right)^{-1} S^{T} A^{T} Q_{y y}^{-1} y \\
& =L^{T} S Q_{\hat{\alpha}_{B} \hat{\alpha}_{B}} S^{T} A^{T} Q_{y y}^{-1} y \\
& =Q_{\hat{z}_{B} \hat{y}_{B}} Q_{y y}^{-1} y
\end{aligned}
$$

with

$Q_{\hat{\hat{z}}_{\hat{Z}_{B}}}=Q_{\hat{\mathrm{z}}_{B} \hat{\vartheta}_{B}} Q_{y y}^{-1} Q_{\hat{y}_{B} \hat{z}_{B}}$

in which use is made of the equalities $Q_{\hat{\alpha}_{\beta} \hat{\alpha}_{B}}=\left(S^{T} N S\right)^{-1}$ and $Q_{\hat{z}_{B} \hat{y}_{B}}=L^{T} S Q_{\hat{\alpha}_{B} \hat{\alpha}_{B}} S^{T} A^{T}$, where $\hat{y}_{B}=A S \hat{\alpha}_{B}$. Using (5), the expectation of $\hat{z}_{B}$ reads
$\mathrm{E}\left(\hat{z}_{B}\right)=Q_{\hat{z}_{B} \hat{y}_{B}} Q_{y y}^{-1} \mathrm{E}(y)=z+h \beta, \quad$ with $\quad h=Q_{\hat{z}_{B} \hat{y}_{B}} Q_{y y}^{-1} \epsilon$

The solution $\hat{z}_{B}$ is thus biased by $h \beta$ when $\beta \neq 0$. Now consider Scenario A. Including the extra parameter $\beta$ into the inconsistent linear system (A.1), i.e.

$y \approx A S \alpha+\epsilon \beta$

the least-squares solution $\hat{\alpha}_{B}$ is adapted to $\hat{\alpha}_{A}$ as follows (Teunissen, 2000)

$\hat{\alpha}_{A}=\hat{\alpha}_{B}-\left(S^{T} N S\right)^{-1} S^{T} N v \hat{\beta}, \quad$ with

$Q_{\hat{\alpha}_{A} \hat{\alpha}_{A}}=Q_{\hat{\alpha}_{B} \hat{\alpha}_{B}}+\sigma_{\hat{\beta}}^{2}\left\{\left(S^{T} N S\right)^{-1} S^{T} N v\right\}\left\{\left(S^{T} N S\right)^{-1} S^{T} N v\right\}^{T}$

From (8), the least-squares solution $\hat{z}_{A}$ follows as

$\hat{z}_{A}=\hat{z}_{B}-h \hat{\beta}, \quad$ with $\quad Q_{\hat{z}_{A} \hat{z}_{A}}=Q_{\hat{z}_{B} \hat{z}_{B}}+\sigma_{\hat{\beta}}^{2} h h^{T}$

The MSE expressions (9) follow then from

Scenario A : $\quad \mathrm{E}\left\|\hat{z}_{A}-z\right\|^{2}=\operatorname{tr}\left(Q_{\hat{z}_{A} \hat{z}_{A}}\right)+\left(\mathrm{E}\left(\hat{z}_{A}\right)-z\right)^{T}\left(\mathrm{E}\left(\hat{z}_{A}\right)-z\right)$

Scenario B : $\quad \mathrm{E}\left\|\hat{z}_{B}-z\right\|^{2}=\operatorname{tr}\left(Q_{\hat{z}_{\hat{B} \hat{B}} \hat{z}_{B}}\right)+\left(\mathrm{E}\left(\hat{z}_{B}\right)-z\right)^{T}\left(\mathrm{E}\left(\hat{z}_{B}\right)-z\right)$

and the equalities $\mathrm{E}\left(\hat{z}_{A}\right)-z=0$ and $\mathrm{E}\left(\hat{z}_{B}\right)-z=h \beta$.

Proof of Lemma 2. Using the equality $c=F^{T} z+b \beta$ and the relation (A.8), one obtains

$\hat{c}_{A}=\hat{c}_{B}-\left(F^{T} h-b\right) \hat{\beta}, \quad$ and $\mathrm{E}\left(\hat{c}_{B}\right)-\mathrm{E}\left(\hat{c}_{A}\right)=\left(F^{T} h-b\right) \beta$

Application of the variance propagation law to $\hat{x}_{u_{A}}=A_{u}^{+}\left(y_{u}+\hat{c}_{A}\right)$, together with $\hat{x}_{u_{B}}=A_{u}^{+}\left(y_{u}+\hat{c}_{B}\right)$, gives then

$$
\begin{aligned}
Q_{\hat{x}_{u_{A}} \hat{x}_{u_{A}}} & =A_{u}^{+}\left(Q_{y_{u} y_{y}}+Q_{\hat{c}_{B} \hat{c}_{B}}+\sigma_{\hat{\beta}}^{2}\left(F^{T} h-b\right)\left(F^{T} h-b\right)^{T}\right) A_{u}^{+T} \\
& =Q_{\hat{x}_{u_{B}} \hat{x}_{u_{B}}}+\sigma_{\hat{\beta}}^{2}\left\{A_{u}^{+}\left(F^{T} h-b\right)\right\}\left\{A_{u}^{+}\left(F^{T} h-b\right)\right\}^{T}
\end{aligned}
$$

The MSE expressions (17) follow from

Scenario A : $\quad \mathrm{E}\left\|F_{u}^{T}\left(\hat{x}_{u_{A}}-x_{u}\right)\right\|^{2}=\operatorname{tr}\left(F_{u}^{T} Q_{\hat{x}_{u_{A}} \hat{x}_{u_{A}}} F_{u}\right)$

$$
+\left\{F_{u}^{T}\left(\mathrm{E}\left(\hat{x}_{u_{A}}\right)-x_{u}\right)\right\}^{T}\left\{F_{u}^{T}\left(\mathrm{E}\left(\hat{x}_{u_{A}}\right)-x_{u}\right)\right\}
$$

Scenario B : $\quad \mathrm{E}\left\|F_{u}^{T}\left(\hat{x}_{u_{B}}-x_{u}\right)\right\|^{2}=\operatorname{tr}\left(F_{u}^{T} Q_{\hat{x}_{u_{B}} \hat{x}_{u_{B}}} F_{u}\right)$

$$
+\left\{F_{u}^{T}\left(\mathrm{E}\left(\hat{x}_{u_{B}}\right)-x_{u}\right)\right\}^{T}\left\{F_{u}^{T}\left(\mathrm{E}\left(\hat{x}_{u_{B}}\right)-x_{u}\right)\right\}
$$

and the equalities $\mathrm{E}\left(\hat{x}_{u_{A}}\right)-x_{u}=0 \quad$ and $\mathrm{E}\left(\hat{x}_{u_{B}}\right)-x_{u}=A_{u}^{+}\left(F^{T} h-b\right) \beta$.

Proof of Eq. (32). Note that (7) can also be expressed as $\overline{\boldsymbol{\epsilon}}=P^{\perp} \boldsymbol{\epsilon}, \quad$ with $\quad P^{\perp}=I-A S\left(S^{T} A^{T} Q_{v y}^{-1} A S\right)^{-1} S^{T} A^{T} Q_{v y}^{-1}$ 
For the specific case (21), both $\epsilon$ and the matrix $P^{\perp}$ are functions of the inter-station distance $d_{1 r}$ through the mapping function $g_{1 r}^{1 s}$. Thus we use the notation $\epsilon\left(d_{1 r}\right)$ and $P^{\perp}\left(d_{1 r}\right)$ to show the stated dependency. Expansion of $\overline{\boldsymbol{\epsilon}}\left(d_{1 r}\right)$ into a Taylor series at the zero inter-station distance $d_{1 r}=0$ gives

$\overline{\boldsymbol{\epsilon}}\left(d_{1 r}\right) \approx \overline{\boldsymbol{\epsilon}}(0)+\dot{\overline{\boldsymbol{\epsilon}}}(0) d_{1 r}$

where $\dot{\bar{\epsilon}}(0)$ is the derivative of $\bar{\epsilon}\left(d_{1 r}\right)$ at $d_{1 r}=0$ and can be computed as follows

$\dot{\overline{\boldsymbol{\epsilon}}}(0)=\dot{P}^{\perp}(0) \boldsymbol{\epsilon}(0)+P^{\perp}(0) \dot{\boldsymbol{\epsilon}}(0)=P^{\perp}(0) \dot{\boldsymbol{\epsilon}}(0), \quad$ since $\quad \boldsymbol{\epsilon}(0)=0$

with $\dot{P}^{\perp}(0)$ and $\dot{\boldsymbol{\epsilon}}(0)$ being the derivatives of $P^{\perp}\left(d_{1 r}\right)$ and $\epsilon(0)$ at $d_{1 r}=0$, respectively. This, together with $\overline{\boldsymbol{\epsilon}}(0)=P^{\perp}(0) \boldsymbol{\epsilon}(0)=0$, gives

$\overline{\boldsymbol{\epsilon}}\left(d_{1 r}\right) \approx P^{\perp}(0) \dot{\boldsymbol{\epsilon}}(0) d_{1 r}$

Substitution into the first expression of (6) gives (32).

\section{References}

Baarda, W., 1981. S-transformations and criterion matrices. In: Publications on geodesy, vol. 5, no. 1, second revised ed. Netherlands Geodetic Commission, Delft, the Netherlands. ISBN-10: 9061322189.

Bevis, M., Businger, S., Herring, T.A., et al., 1992. GPS meteorology: remote sensing of atmospheric water vapor using the global positioning system. J. Geophys. Res. 97 (D14), 15787-15801. https://doi.org/ 10.1029/92JD01517.

Collins, P., 2008. Isolating and estimating undifferenced GPS integer ambiguities. In: Proceedings of the 2008 National Technical Meeting of The Institute of Navigation, San Diego, CA, pp. 720-732.

Dach, R., Lutz, S., Walser, P., et al., 2015. Bernese GNSS Software Version 5.2. User manual. Astronomical Institute, University of Bern, Bern Open Publishing. https://doi.org/10. 7892/boris. 72297.

Dow, J.M., Neilan, R.E., Rizos, C., 2009. The International GNSS service in a changing landscape of global navigation satellite systems. J. Geod. 83 (3-4), 191-198. https://doi.org/10.1007/s00190-008-0300-3.

Ge, M., Gendt, G., Rothacher, M., et al., 2008. Resolution of GPS carrierphase ambiguities in Precise Point Positioning (PPP) with daily observations. J. Geod. 82 (7), 389-399. https://doi.org/10.1007/ s00190-007-0187-4.

Geng, J., Shi, C., 2017. Rapid initialization of real-time PPP by resolving undifferenced GPS and GLONASS ambiguities simultaneously. J. Geod. 91 (4), 361-374. https://doi.org/10.1007/s00190-016-0969-7.

Griffiths, J., Ray, J.R., 2009. On the precision and accuracy of IGS orbits. J. Geod. 83 (3-4), 277-287. https://doi.org/10.1007/s00190008-0237-6.

Hofmann-Wellenhof, B., Lichtenegger, H., Wasle, E., 2008. GNSSGlobal Navigation Satellite Systems: GPS, GLONASS, Galileo, and More. Springer-Verlag, Wien. https://doi.org/10.1007/978-3-21173017-1.

Hoque, M.M., Jakowski, N., 2007. Higher order ionospheric effects in precise GNSS positioning. J. Geod. 81 (4), 259-268. https://doi.org/ 10.1007/s00190-006-0106-0.

Ifadis, I., 1986. The Atmospheric Delay of Radio Waves: Modelling The Elevation Dependence on a Global Scale. Licentiate Thesis. Technical Report No 38L. Chalmers University of Technology, Gothenburg, Sweden.

International GNSS Service (IGS), 2017. GNSS final orbit products. NASA CDDIS. <ftp://cddis.gsfc.nasa.gov/gnss/products/> (accessed July 12, 2017).
Khodabandeh, A., Teunissen, P.J.G., 2015. An analytical study of PPPRTK corrections: precision, correlation and user-impact. J. Geod. 89 (11), 1109-1132. https://doi.org/10.1007/s00190-015-0838-9.

Laurichesse, D., Mercier, F., 2007. Integer ambiguity resolution on undifferenced GPS phase measurements and its application to PPP. In: Proceedings of ION GNSS 2007, Fort Worth, TX, pp. 839-848.

Li, B., Verhagen, S., Teunissen, P.J.G., 2014. Robustness of GNSS integer ambiguity resolution in the presence of atmospheric biases. GPS Solut. 18 (2), 283-296. https://doi.org/10.1007/s10291-013-0329-5.

Liu, Z., Li, Y., Guo, J., et al., 2016. Influence of higher-order ionospheric delay correction on GPS precise orbit determination and precise positioning. Geodesy Geodyn. 7 (5), 369-376. https://doi.org/10.1016/ j.geog.2016.06.005.

Loyer, S., Perosanz, F., Mercier, F., et al., 2012. Zero-difference GPS ambiguity resolution at CNES-CLS IGS analysis center. J. Geod. 86 (11), 991-1003. https://doi.org/10.1007/s00190-012-0559-2.

Mervart, L., Lukes, Z., Rocken, C., et al., 2008. Precise point positioning with ambiguity resolution in real-time. In: Proceedings of ION GNSS 2008, pp. 397-405.

Mousa, A.E.K., Aboualy, N., Sharaf, M., et al., 2016. Tropospheric wet delay estimation using GNSS: case study of a permanent network in Egypt. NRIAG J. Astron. Geophys. 5 (1), 76-86. https://doi.org/ 10.1016/j.nrjag.2016.01.002.

Odijk, D., Arora, B.S., Teunissen, P.J.G., 2014a. Predicting the success rate of long-baseline GPS + Galileo (Partial) ambiguity resolution. J. Navigation 67 (3), 385-401. https://doi.org/10.1017/S037346331400006X.

Odijk, D., Khodabandeh, A., Nadarajah, N., et al., 2017. PPP-RTK by means of S-system theory: Australian network and user demonstration. J. Spatial Sci. 62 (1), 3-27. https://doi.org/10.1080/ 14498596.2016.1261373.

Odijk, D., Teunissen, P.J.G., Khodabandeh, A., 2014b. Single-frequency PPP-RTK: theory and experimental results. In: Rizos, C., Willis, P. (Eds.), Earth on the Edge: Science for a Sustainable Planet. IAG Symposia, vol 139. Springer, Berlin. https://doi.org/10.1007/978-3642-37222-3_75.

Odijk, D., Teunissen, P.J.G., Zhang, B., 2011. PPP-RTK platform performance based on single-frequency GPS data. In: Proceedings of the 32nd Asian Conference on Remote Sensing 2011, ACRS 2011, Oct 3-7, 2011, pp. 2113-2118. Asian Association on Remote Sensing, Taipei, Taiwan.

Odijk, D., Teunissen, P.J.G., Zhang, B., 2012. Single-frequency integer ambiguity resolution enabled GPS precise point positioning. J. Surv. Eng. 138 (4), 193-202. https://doi.org/10.1061/(ASCE)SU.19435428.0000085 .

Odijk, D., Zhang, B., Khodabandeh, A., et al., 2016. On the estimability of parameters in undifferenced, uncombined GNSS network and PPPRTK user models by means of $S$-system theory. J. Geod. 90 (1), 15-44. https://doi.org/10.1007/s00190-015-0854-9.

Resch, G.M., 1984. Water vapor radiometry in geodetic applications. In: Brunner, F.K. (Eds.), Geodetic Refraction. Springer, Berlin, Heidelberg. https://doi.org/10.1007/978-3-642-45583-4_5

Rothacher, M., Beutler, G., 1998. The role of GPS in the study of global change. Phys. Chem. Earth 23 (9-10), 1029-1040. https://doi.org/ 10.1016/S0079-1946(98)00143-8.

Teunissen, P.J.G., 1985a. Zero order design: generalized inverses, adjustment, the datum problem and S-transformations. In: Grafarend, E.W., Sansò, F. (Eds.), Optimization and Design of Geodetic Networks, Springer, Berlin, Heidelberg, pp. 11-55. https://doi.org/10.1007/978-3642-70659-2_3.

Teunissen, P.J.G., 1985b. The geometry of geodetic inverse linear mapping and non-linear adjustment, vol. 8, no. 1. Netherlands Geodetic Commission, Publ. on Geodesy, New Series, Delft, the Netherlands.

Teunissen, P.J.G., 1993. Least-squares estimation of the integer GPS ambiguities. In: Invited Lecture, Section IV Theory and Methodology, IAG General Meeting, Beijing, China, August 1993.

Teunissen, P.J.G., 1995. The least-squares ambiguity decorrelation adjustment: a method for fast GPS integer ambiguity estimation. J. Geod. 70 (1-2), 65-82. https://doi.org/10.1007/BF00863419. 
Teunissen, P.J.G., 2000. Adjustment Theory: an introduction. Series on Mathematical Geodesy and Positioning. VSSD, Delft, The Netherlands.

Teunissen, P.J.G., Khodabandeh, A., 2015. Review and principles of PPPRTK methods. J. Geod. 89 (3), 217-240. https://doi.org/10.1007/ s00190-014-0771-3.

Teunissen, P.J.G., Montenbruck, O. (Eds.) (2017). Springer Handbook of Global Navigation Satellite Systems. Springer International Publishing. https://doi.org/10.1007/978-3-319-42928-1.

Teunissen, P.J.G., Odijk, D., Zhang, B., 2010. PPP-RTK: results of CORS network-based PPP with integer ambiguity resolution. J. Aeronaut. Astronaut. Aviat. 42 (4), 223-230.

Wang, K., Khodabandeh, A., Teunissen, P.J.G., 2017. A study on predicting network corrections in PPP-RTK processing. Adv. Space Res. 60 (7), 1463-1477. https://doi.org/10.1016/j.asr.2017.06.043.
Wang, M., Li, B., 2016. Evaluation of empirical tropospheric models using satellite-tracking tropospheric wet delays with water vapor radiometer at Tongji, China. Sensors 16 (2), 186. https://doi.org/10.3390/ s16020186.

Wen, Z., Henkel, P., Günther, C., 2011. Reliable estimation of phase biases of GPS satellites with a local reference network. ELMAR. In: 2011 Proceedings, September 2011, Zadar, Croatia.

Wübbena, G., Schmitz, M., Bagge, A., 2005. PPP-RTK: precise point positioning using state-space representation in RTK networks. In: Proceedings of ION GNSS, Long Beach, CA, September 2005, pp. 2584-2594

Younes, S.A.M., 2016. Modeling investigation of wet tropospheric delay error and precipitable water vapor content in Egypt. Egypt. J. Remote Sensing Space Sci. 19 (2), 333-342. https://doi.org/10.1016/j. ejrs.2016.05.002. 